TRANSACTIONS OF THE

AMERICAN MATHEMATICAL SOCIETY

Volume 348, Number 3, March 1996

\title{
OPERATOR SEMIGROUP COMPACTIFICATIONS
}

\author{
H. D. JUNGHENN
}

\begin{abstract}
A weakly continuous, equicontinuous representation of a semitopological semigroup $S$ on a locally convex topological vector space $X$ gives rise to a family of operator semigroup compactifications of $S$, one for each invariant subspace of $X$. We consider those invariant subspaces which are maximal with respect to the associated compactification possessing a given property of semigroup compactifications and show that under suitable hypotheses this maximality is preserved under the formation of projective limits, strict inductive limits and tensor products.
\end{abstract}

\section{INTRODUCTION}

Let $U: s \mapsto U_{s}$ be a weakly continuous, equicontinuous representation of a semitopological semigroup $S$ on a locally convex topological vector space $X$. If $Y$ is a $U_{S}$-invariant subspace of $X$ then the weak* operator closure $\mathcal{U}_{Y}$ of $\left.U_{S}\right|_{Y}$ in $\mathcal{L}\left(X, X^{\prime \prime}\right)$ is a left topological semigroup compactification of $S$ under a multiplication which may be viewed as the natural extension of operator composition in $\mathcal{L}(X)$. Given a property $\mathcal{P}$ of semigroup compactifications of $S$ one can ask if there exists a maximal invariant subspace $Y$ of $X$ such that the compactification $\mathcal{U}_{Y}$ has property $\mathcal{P}$. Such a subspace, if it exists, is called a maximal $\mathcal{P}$-subspace of $X$ (or $U$ ). For example, if $U$ is weakly almost periodic and $\mathcal{P}$ is the property that a compactification of $S$ is a group, then the maximal $\mathcal{P}$-subspace of $X$ is the space $X_{\text {sap }}$ of strongly almost periodic vectors as defined by deLeeuw and Glicksberg [3].

In this paper we prove a general existence and uniqueness theorem for maximal $\mathcal{P}$-spaces. We show that for certain properties $\mathcal{P}$, called regular properties, $X$ has a unique maximal $\mathcal{P}$-subspace, which we denote by $X_{\mathcal{P}}$. We then examine the semigroup theoretic and topological dynamical properties of $\mathcal{U}_{X_{\mathcal{P}}}$ as well as the functional analytic properties of $X_{\mathcal{P}}$. We show that under suitable conditions direct products, projective limits, strict inductive limits and tensor products of maximal $\mathcal{P}$-spaces are again maximal $\mathcal{P}$-spaces (relative to the appropriate derived representations) and we characterize the resulting operator semigroup compactifications in terms of those of the given representations. As an illustration of the sort of result that one can expect, consider an infinite family $\left\{X_{i}: i \in I\right\}$ of unital abelian $C^{*}$-algebras and let $X:=\bigotimes_{i \in I} X_{i}$ be the $C^{*}$-algebra tensor product of the $X_{i}$ (see Section 5). For each $i \in I$ let $U^{i}$ be a weakly almost periodic representation of a semitopological semigroup $S_{i}$ by unital *-homomorphisms on $X_{i}$.

Received by the editors October 27, 1994.

1991 Mathematics Subject Classification. Primary 22A20, 22A25, 43A60.

Key words and phrases. Semitopological semigroup, left topological compactification, representation, projective limit, inductive limit, tensor product, weakly almost periodic. 
Let $s \mapsto U_{s}: \prod_{i} S_{i} \mapsto \mathcal{L}(X)$ be the tensor product of the representations $U^{i}$. It follows from general results in Section 5 that $\mathcal{U}_{X}$ is an inverse limit of finite tensor products of the $\mathcal{U}_{X_{i}}^{i}$. Moreover, if $W A P\left(S_{i}\right)$ is left amenable for every $i$ then $X_{\text {sap }}=\bigotimes_{i} X_{\text {isap }}$ and $X$ splits into a direct sum of $X_{\text {sap }}$ and a subspace of flight vectors (see [3]).

\section{Terminology and Notation}

In this section we set down the basic notions of semigroup representations and compactifications needed in the sequel. For details the reader is referred to $[1,2]$.

A semitopological semigroup ( $\mathrm{stsg}$ ) is a semigroup $S$ with a topology relative to which multiplication is separately continuous. If multiplication is jointly continuous then $S$ is a topological semigroup (tsg). If $U: s \mapsto U_{s}$ is a representation of a stsg $S$ on a (separated) locally convex topological vector space (lctvs) $X$ we define the subspaces

$$
\begin{aligned}
X_{w c} & :=\left\{x \in X: s \mapsto U_{s} x \text { is weakly continuous }\right\} \\
X_{a p} & :=\left\{x \in X: U_{S} x \text { is relatively compact }\right\} \\
X_{w a p} & :=\left\{x \in X: U_{S} x \text { is relatively weakly compact }\right\} .
\end{aligned}
$$

If $U$ is equicontinuous (i.e., $U_{S}$ is an equicontinuous family) then $X_{w c}$ and $X_{a p}$ are closed. If, additionally, $X$ is quasicomplete, then $X_{\text {wap }}$ is closed [2, 6.1.14]. $U$ is weakly continuous (weakly almost periodic, almost periodic) if $X=X_{w c}(X=$ $\left.X_{w a p}, X=X_{a p}\right)$. For the right translation representation $s \mapsto R_{s}$ on $C(S)$, the $C^{*}$-algebra of all bounded, continuous, complex-valued functions on $S$, we use the standard notation $W R C(S)=C(S)_{w c}, W A P(S)=C(S)_{w a p}$ and $A P(S)=$ $C(S)_{a p}$.

If $X$ and $Y$ are lctvs then $\mathcal{L}(X, Y)$ denotes the space of continuous linear transformations $u: X \mapsto Y$. The adjoint of $u$ is denoted by $u^{\prime}$. Note that $\mathcal{L}(X)$ $(=\mathcal{L}(X, X))$ is a stsg under composition and the weak operator topology. If $U$ and $V$ are representations of a stsg $S$ on $X$ and $Y$, respectively, and if $u \in \mathcal{L}(X, Y)$ satisfies $u \circ U_{s}=V_{s} \circ u$ for all $s \in S$, then $u\left(X_{w c}\right) \subset Y_{w c}, u\left(X_{w a p}\right) \subset Y_{w a p}$ and $u\left(X_{a p}\right) \subset Y_{a p}$, as is easily verified.

A semigroup $S$ with a topology is left (right) topological if the mappings $\lambda_{t}$ : $s \mapsto t s\left(\rho_{t}: s \mapsto s t\right), t \in S$, are continuous. A compact, Hausdorff, left (or right) topological semigroup $S$ has a smallest ideal $K(S)$, called the minimal ideal of $S$. We use freely the properties of $K(S)$ (see, for example, Sections 1.2 and 1.3 of [2]).

A left topological compactification of a $\operatorname{stsg} S$ is a pair $\left(\varphi, S^{\prime}\right)$, where $S^{\prime}$ is a compact, Hausdorff, left topological semigroup and $\varphi: S \mapsto S^{\prime}$ is a continuous homomorphism with dense range such that the mappings $\rho_{\varphi(s)}: S^{\prime} \mapsto S^{\prime}, s \in S$, are continuous. Right topological compactifications are defined analogously. A compactification $\left(\varphi, S^{\prime}\right)$ is said to be (semi)topological if $S^{\prime}$ is a (semi)topological semigroup. We sometimes omit reference to the mapping $\varphi$ and call $S^{\prime}$ a compactification of $S$. Compactifications in this paper are assumed to be left topological unless otherwise stipulated.

A homomorphism from a compactification $\left(\varphi, S^{\prime}\right)$ of a stsg $S$ to a compactification $\left(\psi, S^{\prime \prime}\right)$ of $S$ is a continuous function $\theta: S^{\prime} \mapsto S^{\prime \prime}$ such that $\theta \circ \varphi=\psi$. The compactification $S^{\prime \prime}$ is then called a factor of $S^{\prime}$ and $S^{\prime}$ an extension of $S^{\prime \prime}$ and we write $\left(\varphi, S^{\prime}\right) \geq\left(\psi, S^{\prime \prime}\right)$. Note that $\theta$ is necessarily unique, surjective and 
a semigroup homomorphism. If $\theta$ is $1-1$ then $\theta^{-1}$ is a compactification homomorphism. In this case $\theta$ is called an isomorphism and we write $\left(\varphi, S^{\prime}\right) \cong\left(\psi, S^{\prime \prime}\right)$. If $\left(\varphi, S^{\prime}\right) \geq\left(\psi, S^{\prime \prime}\right)$ and $\left(\varphi, S^{\prime}\right) ¥\left(\psi, S^{\prime \prime}\right)$ we write $\left(\varphi, S^{\prime}\right)>\left(\psi, S^{\prime \prime}\right)$. The relation $\geq$ partially orders (equivalence classes of) compactifications of $S$.

Let $F$ be a translation invariant subspace of $C(S)$. For $\mu \in F^{\prime}$, the dual space of $F$, define the right introversion operator $\mu_{r}$ on $F$ by $\left(\mu_{r} f\right)(s):=\mu\left(R_{s} f\right) . F$ is right introverted if $\mu_{r} F \subset F$ for every $\mu \in F^{\prime}$. If $F$ is a $C^{*}$-subalgebra of $C(S)$ and $\mu_{r} F \subset F$ for every $\mu \in S^{F}$, the spectrum of $F$, then $F$ is said to be right $m$ introverted. Left introversion and left m-introversion are defined analogously. The space $W R C(S)$ is right introverted [2, 4.5.2], and any closed, translation invariant subspace of $W A P(S)$ is both right and left introverted [2, 4.2.7].

Every left topological compactification $\left(\varphi, S^{\prime}\right)$ of a stsg $S$ is isomorphic to the canonical $F$-compactification $\left(\epsilon, S^{F}\right)$ of $S$. Here, $F$ is the right m-introverted $C^{*}$ subalgebra $\varphi^{*}\left(C\left(S^{\prime}\right)\right)$ (the function space of the compactification), $\epsilon: S \mapsto S^{F}$ is the evaluation mapping $\epsilon(s): f \mapsto f(s)$, and multiplication in $S^{F}$ is given by $\mu * \nu=\mu_{r}^{\prime}(\nu)$. Every translation invariant, right m-introverted $C^{*}$-subalgebra $F$ of $C(S)$ which contains the constant functions is the function space of a left topological compactification of $S$. Similar remarks apply to right topological compactifications.

If $S$ and $T$ are stsgs, $\phi: S \mapsto T$ is a continuous homomorphism and $\left(\psi, T^{\prime}\right)$ is a compactification of $T$, then the induced compactification $(\psi \circ \phi, \overline{\psi \circ \phi(S)})$ of $S$ is called a subcompactification of $\left(\psi, T^{\prime}\right)$. If $\left\{S_{i}: i \in I\right\}$ is a family of stsgs and $\left(\psi_{i}, S_{i}^{\prime}\right)$ is a compactification of $S_{i}$, then the compactification $\left(\prod_{i} \psi_{i}, \prod_{i} S_{i}^{\prime}\right)$ of the stsg $\prod_{i} S_{i}$ is called the direct product of the compactifications $\left(\psi_{i}, S_{i}^{\prime}\right)$. If $S_{i}=S$ for all $i$ and $\psi(s):=\left(\psi_{i}(s)\right)_{i}, s \in S$, then the subcompactification $(\psi, \overline{\psi(S)})$ of $\left(\prod_{i} \psi_{i}, \prod_{i} S_{i}^{\prime}\right)$ is called the subdirect product of the compactifications $\left(\psi_{i}, S_{i}^{\prime}\right)$.

A compactification with a given property $\mathcal{P}$ which is invariant under compactification isomorphisms is called a $\mathcal{P}$-compactification. A universal or maximal $\mathcal{P}$ compactification of a stsg $S$ is a $\mathcal{P}$-compactification which is an extension of every $\mathcal{P}$-compactification of $S$. Minimal $\mathcal{P}$-compactifications are defined analogously. Maximal and minimal $\mathcal{P}$-compactifications of $S$, if they exist, are unique up to isomorphism. We denote the maximal and minimal $\mathcal{P}$-compactifications of $S$ by $S^{\mathcal{P}}$ and $S_{\mathcal{P}}$, respectively.

A property $\mathcal{P}$ of compactifications (of a specified or understood family of stsgs) is said to be inherited by factors (extensions, direct products, subcompactifications, subdirect products) if factors (extensions, direct products, subcompactifications, subdirect products) of $\mathcal{P}$-compactifications are again $\mathcal{P}$-compactifications. For example, let $\mathcal{P}$ be the property of compactifications $\left(\varphi, S^{\prime}\right)$ of a locally compact topological group $S$ that $\lim _{s \rightarrow \infty} \varphi(s)$ exists, and let $\mathcal{Q}$ be the property that $\varphi$ is an isomorphism and $S^{\prime} \backslash \varphi(S)$ is an ideal of $S^{\prime}$. Then $\mathcal{P}$ is inherited by factors, $\mathcal{Q}$ is inherited by extensions and $S^{\mathcal{P}}=S_{\mathcal{Q}}=S^{\infty}$, the one-point compactification of $S$ (with infinity acting as a zero). If $\mathcal{P}$ is, more generally, a property of compact left topological semigroups we say that $\mathcal{P}$ is inherited by continuous homomorphic images if any continuous homomorphic image of a compact left topological semigroup with property $\mathcal{P}$ also has property $\mathcal{P}$. Similarly, $\mathcal{P}$ is inherited by direct products if the direct product of a family of compact left topological semigroups with property $\mathcal{P}$ also has property $\mathcal{P}$.

We shall call a property $\mathcal{P}$ of compactifications (resp., compact left topological semigroups) regular if universal $\mathcal{P}$-compactifications exist and if $\mathcal{P}$ is inherited by 
factors (resp., continuous homomorphic images). Note that the conjunction of regular properties is regular. Some examples of regular properties are: the property of being a topological or semitopological semigroup or group, the property of being a semilattice, the property of being a left simple, right simple or simple semigroup, the property of having a left, right or two-sided zero, and the property that the minimal ideal is left simple or right simple. These are also examples of properties which are inherited by direct products.

\section{Maximal $\mathcal{P}$-Subspaces of a Representation}

Let $X$ be a lctvs with strong dual $X^{\prime}=X_{\beta}^{\prime}$ and let $X^{\prime \prime}=\left(X_{\beta}^{\prime}\right)^{\prime}$ be the bidual of $X$ with the (natural) topology of uniform convergence on equicontinuous subsets of $X^{\prime}$. We identify $X$ with its image in the bidual under the canonical imbedding $x \mapsto \hat{x}$. The topology of $X^{\prime \prime}$ is generated by the seminorms

$$
q_{A^{\prime}}\left(x^{\prime \prime}\right):=\sup \left\{\left|\left\langle x^{\prime}, x^{\prime \prime}\right\rangle\right|: x^{\prime} \in A^{\prime}\right\}
$$

where $A^{\prime}$ is an equicontinuous subset of $X^{\prime}$. The topology of $X$ is generated by the seminorms $p_{A^{\prime}}:=\left.q_{A^{\prime}}\right|_{X}$.

Following Witz [12] we define the product $u v$ of $u, v \in \mathcal{L}\left(X, X^{\prime \prime}\right)$ by $u v:=\tilde{u}^{\prime} \circ v$, where $\tilde{u}:=\left.u^{\prime}\right|_{X^{\prime}} \in \mathcal{L}\left(X^{\prime}\right)$. Thus

$$
\left\langle x^{\prime}, u v(x)\right\rangle=\left\langle u^{\prime}\left(x^{\prime}\right), v(x)\right\rangle \quad\left(x \in X, x^{\prime} \in X^{\prime}\right) .
$$

Under this multiplication, $\mathcal{L}\left(X, X^{\prime \prime}\right)$ is a left topological semigroup in the weak* operator topology, and the mapping $u \mapsto \tilde{u}: \mathcal{L}\left(X, X^{\prime \prime}\right) \mapsto \mathcal{L}\left(X^{\prime}\right)$ is a homeomorphism and a semigroup anti-isomorphism. If $v(x) \in X$ then $(u v)(x)=u(v(x))$, hence multiplication restricted to $\mathcal{L}\left(X, X^{\prime \prime}\right) \times \mathcal{L}(X)$ is separately continuous. In particular, $\mathcal{L}(X)$ is a semitopological subsemigroup of $\mathcal{L}\left(X, X^{\prime \prime}\right)$.

Now let $U$ be an equicontinuous representation of a stsg $S$ on $X$. For a $U$ invariant subspace $Y$ of $X$ let $\mathcal{U}_{Y}$ denote the closure of $\left.U_{S}\right|_{Y}$ in the weak* operator topology of $\mathcal{L}\left(Y, Y^{\prime \prime}\right)$. Then $\mathcal{U}_{Y}$ is equicontinuous and is a weak* operator compact subsemigroup of $\mathcal{L}\left(Y, Y^{\prime \prime}\right)$. Moreover, $\mathcal{U}_{Y} \subset \mathcal{L}(Y)$ if and only if $Y \subset X_{\text {wap }}$, in which case $\mathcal{U}_{Y}$ is a stsg and is the weak operator closure of $\left.U_{S}\right|_{Y}$ in $\mathcal{L}(Y)$. If $Y \subset X_{a p}$ then $\mathcal{U}_{Y}$ is a topological semigroup, and if $Y \subset X_{w c}$ then $\mathcal{U}_{Y}$ is a left topological compactification of $S$ (with compactification map $\left.s \mapsto U_{s}\right|_{Y}$ ). We write $\mathcal{U}$ for $\mathcal{U}_{X}$. Compactifications of semigroups of operators on Banach spaces were studied by deLeeuw and Glicksberg $[3,4]$ in the weakly almost periodic case and by K. Witz in the general case in connection with various ergodic problems [12].

The following simple result will have important consequences in what follows.

Lemma 1. Let $U$ be a weakly continuous, equicontinuous representation of a stsg $S$ on a lctvs $X$. If $Y$ is a $U$-invariant subspace of $X$ then $\mathcal{U} \geq \mathcal{U}_{Y}$. The compactification homomorphism $\pi_{Y X}: \mathcal{U} \mapsto \mathcal{U}_{Y}$ satisfies

$$
\left\langle\left. x^{\prime}\right|_{Y}, \pi_{Y X}(u)(y)\right\rangle=\left\langle x^{\prime}, u(y)\right\rangle \quad\left(u \in \mathcal{U}, y \in Y, x^{\prime} \in X^{\prime}\right) .
$$

Proof. The mapping $\pi_{Y X}$ defined by (1) is well defined, continuous, and maps $U_{s}$ to $\left.U_{s}\right|_{Y}$. Therefore $\pi_{Y X}$ is a compactification homomorphism, hence $\mathcal{U} \geq \mathcal{U}_{Y}$.

Note that $\mathcal{U}_{Y}$ is isomorphic to $\left.\mathcal{U}\right|_{Y}:=\left\{\left.u\right|_{Y}: u \in \mathcal{U}\right\} \subset \mathcal{L}\left(Y, X^{\prime \prime}\right)$, where multiplication in $\left.\mathcal{U}\right|_{Y}$ is defined by $\left.\left.u\right|_{Y} v\right|_{Y}=\left.(u v)\right|_{Y}$. Indeed, the mapping $\left.\pi_{Y X}(u) \mapsto u\right|_{Y}$ is easily seen to be a compactification isomorphism. 
Example 1. Consider the right translation representation $s \mapsto R_{s}$ on the Banach space $X:=W R C(S)$. Let $Y$ be a right translation invariant subspace of $X$. Denote by $Y^{r}$ the right introverted subspace generated by $Y$ and let $Y^{a}$ denote the (necessarily right m-introverted) $C^{*}$-algebra generated by $Y^{r}$ and the constant functions. We use the compactification homomorphism of Lemma 1 to show that

(a) $\mathcal{R}_{Y} \cong \mathcal{R}_{Y^{r}}$

(b) $\mathcal{R}_{Y} \cong S^{Y^{a}}$ if $S$ has a right identity and $Y^{a} \subset W R C(S)$.

(The inclusion $Y^{a} \subset W R C(S)$ in (b) holds if $S$ is a $k$-space or a complete metric semitopological group, since in each case $W R C(S)$ is an algebra [2, p. 173].)

To verify (a) note first that for $\mu, \nu \in Y^{r \prime}, s \in S$ and $f \in Y^{r}$ we have $\left\langle\nu, R_{s}\left(\mu_{r} f\right)\right\rangle=\left\langle R_{s} f, \mu_{r}^{\prime}(\nu)\right\rangle$, so letting $R_{s}$ tend to $u$ in $\mathcal{R}_{Y^{r}}$ we obtain

$$
\left\langle\nu, u\left(\mu_{r} f\right)\right\rangle=\left\langle\mu_{r}^{\prime}(\nu), u(f)\right\rangle \quad\left(\mu, \nu \in Y^{r^{\prime}}, u \in \mathcal{R}_{Y^{r}}, f \in Y^{r}\right) .
$$

If $f \in Y$, then the right side of $(2)$ is $\left\langle\left.\mu_{r}^{\prime}(\nu)\right|_{Y}, \pi_{Y^{r}}(u)(f)\right\rangle$, which shows that $\pi_{Y Y^{r}}$ is 1-1 and therefore a compactification isomorphism.

To verify (b) define $\theta: \mathcal{R}_{Y^{r}} \mapsto S^{Y^{a}}$ by

$$
\theta\left(\pi_{Y^{r} Y^{a}}(u)\right)(f)=\left\langle\epsilon\left(1_{r}\right), u(f)\right\rangle \quad\left(u \in \mathcal{R}_{Y^{a}}, f \in Y^{a}\right)
$$

where $1_{r}$ is a right identity of $S$. $\theta$ is well-defined, for if $\pi_{Y^{r} Y^{a}}(u)=\pi_{Y^{r} Y^{a}}(v), u, v \in$ $\mathcal{R}_{Y^{a}}$, then, since $u$ and $v$ are weak* operator limits of homomorphisms, $\langle\nu, u(f g)\rangle=$ $\langle\nu, v(f g)\rangle$ for all $f, g \in Y^{r}$ and all $\nu \in S^{Y^{a}}$, hence $\left\langle\epsilon\left(1_{r}\right), u(h)\right\rangle=\left\langle\epsilon\left(1_{r}\right), v(h)\right\rangle$ for all $h \in Y^{a}$. Since $\theta$ is continuous and $\theta\left(R_{s}\right)=\epsilon(s), \theta$ is a compactification homomorphism. Moreover, for $f \in Y^{r}$ and $v \in \mathcal{R}_{Y^{r}}, \theta(v)(f)=\left\langle\left.\epsilon\left(1_{r}\right)\right|_{Y^{r}}, v(f)\right\rangle$ from (1), hence, from (2), $\theta(v)\left(\mu_{r} f\right)=\left\langle\mu *\left(\left.\epsilon\left(1_{r}\right)\right|_{Y^{r}}\right), v(f)\right\rangle=\langle\mu, v(f)\rangle$ for all $\mu \in Y^{r^{\prime}}$. Therefore $\theta$ is $1-1$ and hence is an isomorphism.

Definition 1. Let $U$ be an equicontinuous representation of a stsg $S$ on a lctvs $X$ and let $\mathcal{P}$ be a property of compactifications of $S$. A $U$-invariant subspace $Y$ of $X$ is called a $\mathcal{P}$-subspace of $X$ (or of the representation $U$ ) if the compactification $\mathcal{U}_{Y}$ has property $\mathcal{P}$. A $\mathcal{P}$-subspace of $X$ which is not properly contained in another $\mathcal{P}$-subspace is called a maximal $\mathcal{P}$-subspace of $X$. If $X$ is a $\mathcal{P}$-space then $U$ is called a $\mathcal{P}$-representation of $S$.

Note that the closure of a $\mathcal{P}$-subspace is a $\mathcal{P}$-subspace $\left(\pi_{Y \bar{Y}}\right.$ is a compactification isomorphism).

The following example shows that a representation need not have a maximal $\mathcal{P}$-space.

Example 2. Let $S=(\mathbb{R},+), X=W R C(S), U_{s}=R_{s}$, and let $\mathcal{P}$ be the property of compactifications $\left(\varphi, S^{\prime}\right)$ of $S$ that $\varphi$ is not 1-1. For $p>0$ let $Y_{p}$ be the space of all periodic functions in $X$ with $p$ as a period. An invariant subspace $Y$ of $X$ is a $\mathcal{P}$-subspace if and only if $Y \subset Y_{p}$ for some $p>0$. Since $Y_{p}$ is a proper subset of $Y_{2 p}$, a maximal $\mathcal{P}$-subspace cannot exist. Note that if $\mathcal{Q}$ is the negation of $\mathcal{P}$ (namely, the property that $\varphi$ is $1-1$ ), then $X$ is the unique maximal $\mathcal{Q}$-subspace, and each nonperiodic function in $X$ generates a minimal $\mathcal{Q}$-subspace (analogously defined).

The next example shows that maximal $\mathcal{P}$-spaces need not be unique. 
Example 3. Let $\mathcal{P}$ be the property of compactifications $\left(\varphi, S^{\prime}\right)$ of a stsg $S$ that $\varphi$ is constant on a nontrivial subgroup of $S$. If $X=W R C(S)$ and $U_{s}=R_{s}$ then an invariant subspace $Y$ of $X$ is a $\mathcal{P}$-subspace if and only if there exists a nontrival subgroup $T$ of $S$ such that $Y \subset Y_{T}:=\left\{f \in X: f(s t)=f\left(s t^{\prime}\right)\right.$ for all $t, t^{\prime} \in T, s \in$ $S\}$. Let $S$ be a discrete, commutative semigroup which is the disjoint union of nontrivial simple subgroups $S_{1}$ and $S_{2}$ such that $s_{1} s_{2}=s_{2}, s_{i} \in S_{i}$. Then $S_{1}$ and $S_{2}$ are the only nontrivial subgroups of $S$, and since $Y_{S_{j}}=\left\{f:\left.f\right|_{S_{j}}\right.$ is constant $\}$ it follows that $Y_{S_{1}}$ and $Y_{S_{2}}$ are distinct maximal $\mathcal{P}$-subspaces of $X$.

For regular properties, the pathologies exhibited by Examples 2 and 3 cannot occur. Indeed, if $\mathcal{P}$ is regular then the union of all $\mathcal{P}$-subspaces of $X$ is a $\mathcal{P}$-space. To prove this we use the first part of the following lemma.

Lemma 2. Let I be a directed set and let $\left\{\left(\psi_{i}, S_{i}^{\prime}\right): i \in I\right\}$ be a family of compactifications of a stsg $S$ such that $S_{i}^{\prime} \leq S_{j}^{\prime}$ whenever $i \leq j$. Let

$$
S^{\prime}:=\lim _{\longleftarrow} S_{i}^{\prime}=\left\{\left(u_{i}\right)_{i} \in \prod_{i \in I} S_{i}^{\prime}: \theta_{i j}\left(u_{j}\right)=u_{i}, i \leq j\right\}
$$

be the inverse limit of the semigroups $S_{i}^{\prime}$ relative to the compactification homomorphisms $\theta_{i j}: S_{j}^{\prime} \mapsto S_{i}^{\prime}, i \leq j$, and set $\psi(s):=\left(\psi_{i}(s)\right)_{i}, s \in S$. Then $\left(\psi, S^{\prime}\right)$ is the compactification of $S$ which is minimal with respect to the property that $\left(\psi, S^{\prime}\right) \geq\left(\psi_{i}, S_{i}^{\prime}\right)$ for all $i$. Moreover, $K\left(S^{\prime}\right)=\lim _{\longleftarrow} K\left(S_{i}^{\prime}\right)$ (relative to the mappings $\left.\left.\theta_{i j}\right|_{K\left(S_{j}^{\prime}\right)}\right)$.

Proof. Let $\left(\phi, S^{\prime \prime}\right)$ be an extension of every $\left(\psi_{i}, S_{i}^{\prime}\right)$ and let $\theta_{i}: S^{\prime \prime} \mapsto S_{i}^{\prime}$ be the corresponding compactification homomorphism. Define $\theta: S^{\prime \prime} \mapsto \prod_{i} S_{i}^{\prime}$ by $\theta(u)=$ $\left(\theta_{i}(u)\right)_{i}$. We claim that $\theta\left(S^{\prime \prime}\right)=S^{\prime}$. Indeed, if $i \leq j$ then $\theta_{i j} \circ \theta_{j}=\theta_{i}$, hence $\theta\left(S^{\prime \prime}\right) \subset S^{\prime}$. For the reverse inclusion let $\left(u_{i}\right)_{i} \in S^{\prime}$ and note that since $I$ is directed and $\theta_{i}$ is surjective, the sets $\theta_{i}^{-1}\left(u_{i}\right), i \in I$, have the finite intersection property and hence must contain a common point $u \in \theta^{-1}\left(\left(u_{i}\right)_{i}\right)$. Therefore $\theta$ maps $S^{\prime \prime}$ continuously onto $S^{\prime}$. Since $\theta \circ \phi=\psi$ it follows that $\left(\psi, S^{\prime}\right)$ is a compactification of $S$ and a factor of $\left(\phi, S^{\prime \prime}\right)$.

To prove the last assertion of the lemma, observe that the inverse limit $J:=$ $\lim _{\longleftarrow} K\left(S_{i}^{\prime}\right)$ is defined since $\theta_{i j}\left(K\left(S_{j}^{\prime}\right)\right)=K\left(S_{i}^{\prime}\right), i \leq j$. Let $p_{i}: S^{\prime} \mapsto S_{i}^{\prime}$ be the projection homomorphism. Since $p_{i}\left(S^{\prime}\right)=S_{i}^{\prime}, p_{i}\left(K\left(S^{\prime}\right)\right)=K\left(S_{i}^{\prime}\right)$. Therefore $J$ contains the idempotents of $K\left(S^{\prime}\right)$ so $J$ is nonempty and hence is an ideal containing $K\left(S^{\prime}\right)$. If $s^{\prime}=\left(s_{i}^{\prime}\right)_{i} \in J$ then for each $i$ there exists a unique idempotent $e_{i} \in K\left(S_{i}^{\prime}\right)$ such that $s_{i}^{\prime} \in e_{i} S_{i}^{\prime} e_{i}$. If $i \leq j$ then $d_{i}:=\theta_{i j}\left(e_{j}\right)$ is an idempotent in $K\left(S_{i}^{\prime}\right)$ and $s_{i}^{\prime}=\theta_{i j}\left(s_{j}^{\prime}\right)=\theta_{i j}\left(e_{j} s_{j}^{\prime} e_{j}\right)=d_{i} s_{i}^{\prime} d_{i}$ so $e_{i}=d_{i}$. Therefore $e=\left(e_{i}\right)_{i} \in S^{\prime}$ and $e s^{\prime} e=s^{\prime}$. Thus, to show that $s^{\prime} \in K\left(S^{\prime}\right)$ it suffices to show that $e \in K\left(S^{\prime}\right)$. This follows easily from the characterization of idempotents in the minimal ideal of a semigroup as minimal relative to the natural partial ordering of idempotents (see, for example, $[2,1.2 .29])$.

The compactification $\left(\psi, S^{\prime}\right)$ of Lemma 2 is called the inverse limit of the compactifications $\left(\psi_{i}, S_{i}^{\prime}\right)$ and is denoted by $\lim _{\longleftarrow}\left(\psi_{i}, S_{i}^{\prime}\right)$ (or simply by $\lim _{\longleftarrow} S_{i}^{\prime}$ ).

Corollary 1. If $\mathcal{P}$ is a regular property of compactifications and if each $\left(\psi_{i}, S_{i}^{\prime}\right)$ is a $\mathcal{P}$-compactification of $S$, then $\lim \left(\psi_{i}, S_{i}^{\prime}\right)$ is a $\mathcal{P}$-compactification of $S$. 
Corollary 2. Let $U$ be a weakly continuous, equicontinuous representation of a stsg $S$ on a lctvs $X$, let $I$ be a directed set and let $\left\{Y_{i}: i \in I\right\}$ be a family of $U$-invariant subspaces of $X$ such that $Y_{i} \subset Y_{j}$ whenever $i \leq j$. Then $Y:=\bigcup_{i \in I} Y_{i}$ is a $U$-invariant subspace of $X$ and $\mathcal{U}_{Y} \cong \lim _{\mathcal{U}_{Y_{i}}}$. Hence, if $\mathcal{P}$ is a regular property of compactifications and each $Y_{i}$ is a $\mathcal{P}$-space, then $Y$ is a $\mathcal{P}$-space.

Proof. $Y$ is clearly a $U$-invariant subspace of $X$ and $\mathcal{U}_{Y} \geq \mathcal{U}_{Y_{i}}$ for all $i$. By Lemma 2, $\mathcal{U}_{Y} \geq \lim \mathcal{U}_{Y_{i}}$ under the compactification homomorphism $\Psi: \mathcal{U}_{Y} \mapsto \prod_{i \in I} \mathcal{U}_{Y_{i}}$, $u \mapsto\left(\pi_{Y_{i} Y}(u)\right)_{i}$. It follows from (1) that $\Psi$ is $1-1$ and hence is an isomorphism. The last assertion of the corollary is a consequence of Corollary 1.

Theorem 1. Let $U$ be a weakly continuous, equicontinuous representation of a stsg $S$ on a lctvs $X$. If $\mathcal{P}$ is a regular property of compactifications then $X$ has a unique maximal $\mathcal{P}$-subspace $X_{\mathcal{P}}$. Moreover, $X_{\mathcal{P}}$ is closed and the $\mathcal{P}$-subspaces of $X$ are precisely the invariant subspaces of $X_{\mathcal{P}}$.

Proof. We show first that if $Y$ and $Z$ are $\mathcal{P}$-subspaces then so is $W:=Y+Z$. Let $S^{\prime}$ be the universal left topological compactification of $S$ and consider the compactification homomorphisms $\phi: S^{\prime} \mapsto S^{\mathcal{P}}, \phi_{W}: S^{\prime} \mapsto \mathcal{U}_{W}, \theta_{Y}: S^{\mathcal{P}} \mapsto \mathcal{U}_{Y}$ and $\theta_{Z}: S^{\mathcal{P}} \mapsto \mathcal{U}_{Z}$. Define $\theta_{W}: S^{\mathcal{P}} \mapsto \mathcal{U}_{W}$ by $\theta_{W}(\phi(t))=\phi_{W}(t), t \in S^{\prime} . \theta_{W}$ is well defined, since if $w^{\prime} \in W^{\prime}, y \in Y$ and $z \in Z$ then from (1)

$$
\begin{aligned}
\left\langle w^{\prime}, \phi_{W}(t)(y+z)\right\rangle & =\left\langle w^{\prime}, \phi_{W}(t)(y)\right\rangle+\left\langle w^{\prime}, \phi_{W}(t)(z)\right\rangle \\
& =\left\langle\left. w^{\prime}\right|_{Y},\left(\pi_{Y W} \circ \phi_{W}\right)(t)(y)\right\rangle+\left\langle\left. w^{\prime}\right|_{Z},\left(\pi_{Z W} \circ \phi_{W}\right)(t)(z)\right\rangle \\
& =\left\langle\left. w^{\prime}\right|_{Y},\left(\theta_{Y} \circ \phi\right)(t)(y)\right\rangle+\left\langle\left. w^{\prime}\right|_{Z},\left(\theta_{Z} \circ \phi\right)(t)(z)\right\rangle .
\end{aligned}
$$

Since $\theta_{W}$ is obviously a compactification homomorphism, the regularity of $\mathcal{P}$ implies that $W$ is a $\mathcal{P}$-subspace of $X$.

Now let $\mathcal{Y}$ denote the family of all $\mathcal{P}$-subspaces of $X$. Since the trivial semigroup is a $\mathcal{P}$-compactification of $S$ (it is a homomorphic image of $S^{\mathcal{P}}$ ), $\{0\}$ is a $\mathcal{P}$-subspace of $X$, so $\mathcal{Y} \neq \emptyset$. By the first part of the proof, $\mathcal{Y}$ is directed upward by inclusion. Therefore, by Corollary 2 of Lemma $2, X_{\mathcal{P}}:=\bigcup \mathcal{Y}$ is a $\mathcal{P}$-subspace of $X$. Since $\bar{X}_{\mathcal{P}}$ is also a $\mathcal{P}$-subspace, $X_{\mathcal{P}}$ is closed. That any invariant subspace of $X_{\mathcal{P}}$ is a $\mathcal{P}$-space is consequence of Lemma 1 and the regularity of $\mathcal{P}$.

Theorem 2. Let $U$ be a weakly continuous representation of a stsg $S$ on a commutative $C^{*}$-algebra $X$. If each $U_{s}$ is a ${ }^{*}$-homomorphism and if either $U_{S}$ is weakly almost periodic or $X$ has separable dual, then $X_{\mathcal{P}}$ is a $C^{*}$-subalgebra of $X$. If $X$ and the operators $U_{s}$ are unital then $X_{\mathcal{P}}$ is unital.

Proof. Let $\Omega(X)$ denote the spectrum of $X$ with the weak* topology. Since $u \in \mathcal{U}$ is the weak* operator limit of $*$-homomorphisms we have

$$
\langle\tau, u(x y)\rangle=\langle\tau, u(x)\rangle\langle\tau, u(y)\rangle \quad(x, y \in X, \tau \in \Omega(X))
$$

and

$$
\left\langle\tau, u\left(x^{*}\right)\right\rangle=\overline{\left\langle\tau^{*}, u(x)\right\rangle} \quad\left(x \in X, \tau \in X^{\prime}\right),
$$

where $\tau^{*}(x):=\overline{\tau\left(x^{*}\right)}$. Let $Y=X_{\mathcal{P}}$ and let $Z$ be the (necessarily $U_{S}$-invariant) $C^{*}$-algebra generated by $Y$. To show that $Y=Z$ it suffices to show that the 
compactification homomorphism $\pi_{Y Z}: \mathcal{U}_{Z} \mapsto \mathcal{U}_{Y}$ is 1-1. A typical member of $Z$ is a limit of sums

$$
z=\sum_{i} x_{i 1} x_{i 2} \cdots x_{i n_{i}} y_{i 1}^{*} y_{i 2}^{*} \cdots y_{i m_{i}}^{*} \quad\left(x_{i k}, y_{i k} \in Y\right) .
$$

For any $\tau \in \Omega(Z)$ and $u \in \mathcal{U}_{Z}$ we have, by (1), (3) and (4),

$$
\begin{aligned}
\langle\tau, u(z)\rangle & =\sum_{i} \prod_{j}\left\langle\tau, u\left(x_{i j}\right)\right\rangle \prod_{k} \overline{\left\langle\tau^{*}, u\left(y_{i k}\right)\right\rangle} \\
& =\sum_{i} \prod_{j}\left\langle\left.\tau\right|_{Y}, \pi_{Y Z}(u)\left(x_{i j}\right)\right\rangle \prod_{k} \overline{\left\langle\left.\tau^{*}\right|_{Y}, \pi_{Y Z}(u)\left(y_{i k}\right)\right\rangle} .
\end{aligned}
$$

Now let $u, v \in \mathcal{U}_{Z}$ with $\pi_{Y Z}(u)=\pi_{Y Z}(v)$. From (5),

$$
\langle\tau, u(z)\rangle=\langle\tau, v(z)\rangle \quad(\tau \in \Omega(Z), z \in Z) .
$$

We claim that $u=v$. If $U_{S}$ is weakly almost periodic this follows from (6) and the Gelfand representation. Now suppose that $X$ has separable dual and let $z \in$ $Z$. Then $\mathcal{U}(z)$ is weak* metrizable hence there exist sequences $U_{s_{n}} z \stackrel{w^{*}}{\rightarrow} u(z)$ and $U_{t_{n}} z \stackrel{w^{*}}{\rightarrow} v(z)$. From $(6)$ we have $\left\langle\left(U_{s_{n}}-U_{t_{n}}\right)(z), \tau\right\rangle \rightarrow 0, \tau \in \Omega(Z)$. Since an arbitrary member $z^{\prime}$ of $Z^{\prime}$ can be represented by a Borel measure on $\Omega(Z)$, the dominated convergence theorem implies that $\left\langle z^{\prime}, u(z)-v(z)\right\rangle=0$ so $u=v$ in the separable case as well. Therefore $Z=X_{\mathcal{P}}$.

If $X$ and the operators $U_{s}$ are unital and if $W$ denotes the $C^{*}$-algebra generated by the unit and $X_{\mathcal{P}}$, then $\pi_{X_{\mathcal{P}} W}$ is an isomorphism hence $W=X_{\mathcal{P}}$.

If $U$ is a weakly continuous, equicontinuous representation of a stsg $S$ on a lctvs $X$ then by Theorem 1 there exist maximal subspaces $X_{s t s g}$ and $X_{t s g}$ of $X$ such that $\mathcal{U}_{X_{s t s g}}$ is a stsg and $\mathcal{U}_{X_{t s g}}$ is a tsg. By maximality,

$$
X_{w a p} \subset X_{\text {stsg }} \quad \text { and } \quad X_{a p} \subset X_{t s g}
$$

We make use of these spaces in the following examples.

Example 4. Consider the right translation representation $R$ on the space $X=$ $W R C(S)$. If $S$ has a topological left (or right) identity then equality holds in (7). Indeed, for sequences $\left\{R_{s_{n}}\right\}$ and $\left\{R_{t_{m}}\right\}$ and for $f \in W R C(S)_{\text {stsg }}$ and $\tau \in$ $W R C(S)^{\prime}$, the separate continuity of multiplication in $\mathcal{R}_{W R C(S)_{s t s g}}$ implies that $\lim _{n} \lim _{m}\left\langle R_{s_{n}} R_{t_{m}} f, \tau\right\rangle=\lim _{m} \lim _{n}\left\langle R_{s_{n}} R_{t_{m}} f, \tau\right\rangle$ whenever all limits exist. Taking $\tau$ to be a weak* limit point of the net of evaluations at the topological left identity of $S$ gives $\lim _{n} \lim _{m} f\left(s_{n} t_{m}\right)=\lim _{m} \lim _{n} f\left(s_{n} t_{m}\right)$, which is the double limit criterion for weak almost periodicity of $f$. Thus $W A P(S)=W R C(S)_{\text {stsg }}$. To see that $A P(S)=W R C(S)_{t s g}$, let $f \in W R C(S)_{t s g}$ and let $R_{s_{\alpha}} \rightarrow u$ in $\mathcal{R}_{W R C(S)_{t s g}}$. The joint continuity of multiplication in $\mathcal{R}_{W R C(S)_{t s g}}$ implies that $R_{s_{\alpha}} f(t)=\left\langle\tau, R_{t} R_{s_{\alpha}} f\right\rangle$ converges uniformly in $t$ to $\left\langle\tau, R_{t} u f\right\rangle$, hence $f \in A P(S)$.

The inclusions in (7) can be strict. For example, let $S=[0, \infty)^{3}$ with the usual topology and with multiplication $(a, b, c)\left(a^{\prime}, b^{\prime}, c^{\prime}\right)=\left(0, a c^{\prime}, 0\right)$. For any 
$f \in W R C(S)$ and $s, t \in S, R_{s} R_{t} f$ is the constant function $f(0,0,0)$ hence $(u v)(f)=$ $f(0,0,0)$ for all $u, v \in \mathcal{R}_{W R C(S)}$. Therefore, trivially, $W R C(S)_{\text {stsg }}=W R C(S)_{t s g}$ $=W R C(S)$. But it is easy to construct functions $f$ in $W R C(S) \backslash W A P(S)$. For example, if $g(a)=\arctan (\ln a), a>0$, and $g(0)=-\pi / 2$, then $f(a, b, c)=g(b)$ is such a function.

In the next examples we consider maximal $\mathcal{P}$-spaces which arise from certain topological dynamics considerations. These are based on the observation that for each $x \in X$ the semigroup $S$ acts on $\mathcal{U}(x)$ by

$$
(s, u(x)) \mapsto\left(U_{s} u\right)(x),
$$

the action being jointly continuous if $x \in X_{t s g}$ and separately continuous if $S$ is commutative or if $x \in X_{\text {sts }}$. In the latter case the map

$$
\theta_{x}: \mathcal{U} \mapsto \Sigma_{x}, \quad \theta_{x}(u)(v(x))=u v(x)
$$

is a compactification homomorphism, where $\Sigma_{x}$ is the enveloping semigroup of the action.

Example 5. Call a vector $x \in X$ distal (with respect to the representation $U$ ) if the action in (8) is distal, i.e., if $\lim _{\alpha}\left(U_{s_{\alpha}} u\right)(x)=\lim _{\alpha}\left(U_{s_{\alpha}} v\right)(x)$ implies $u(x)=v(x)$. Denote the set of all distal vectors by $X_{d}$. We show that if $x \in X_{\text {stsg }}$ then the following statements are equivalent:

(a) $x \in X_{d}$.

(b) $u, u_{1}, u_{2} \in \mathcal{U}$ and $u u_{1}(x)=u u_{2}(x) \Rightarrow u_{1}(x)=u_{2}(x)$.

(c) $e v(x)=v(x)$ for all (minimal) idempotents $e \in \mathcal{U}$ and all $v \in \mathcal{U}$.

The equivalence of (a) and (b) is an obvious consequence of the separate continuity of multiplication in $\mathcal{U}_{X_{s t s g}}$. That $(\mathrm{b}) \Rightarrow(\mathrm{c})$ is seen by taking $u=e, u_{1}=e v$ and $u_{2}=v$ in (b), where $e$ is an arbitrary idempotent in $\mathcal{U}$. For (c) $\Rightarrow$ (a) let $e \in \mathcal{U}$ be a minimal idempotent (i.e., an idempotent in $K(\mathcal{U})$ ) such that $e v(x)=v(x)$ for all $v \in \mathcal{U}$. Then $\theta_{x}(e)$ is the identity of $\Sigma_{x}$, hence $\Sigma_{x}=\theta_{x}(\mathcal{U})=\theta_{x}(e \mathcal{U} e)$. Since $e \mathcal{U} e$ is a group, it follows from a well known theorem of Ellis $[5,6]$ that $x \in X_{d}$.

If $S$ is commutative then similar arguments show that for arbitrary $x \in X$, $x \in X_{d}$ iff $e v(x)=v e(x)=v(x)$ for all $e^{2}=e, v \in \mathcal{U}_{X_{d}}$.

It follows from (c) that $X_{\text {stsg }} \cap X_{d}$ is a closed, invariant subspace of $X$ and is maximal with respect to the property that $\mathcal{U}_{X_{s t s g} \cap X_{d}}$ is a right simple stsg compactification of $S$. Similarly, if $S$ is commutative then $X_{d}$ is a closed, invariant subspace of $X$ and is maximal with respect to the property that $\mathcal{U}_{X_{d}}$ is a left topological group.

Example 6. Call a vector $x \in X$ minimal (with respect to $U$ ) if for each $v \in \mathcal{U}$ there exists $u \in \mathcal{U}$ such that $u v(x)=x$. Denote the set of all minimal vectors by $X_{m}$ and set $X_{m d}:=X_{m} \cap X_{d}$. We show that for $x \in X_{\text {stsg }}$,

(a) $x \in X_{m} \Longleftrightarrow$ there exists a minimal idempotent $e \in \mathcal{U}$ such that $e(x)=x$;

(b) $x \in X_{m d} \Longleftrightarrow e v(x)=v(x)$ and $e(x)=x$ for all $v \in \mathcal{U}$ and all (minimal) idempotents $e \in \mathcal{U}$.

(Part (a) is proved in [3] for weakly almost periodic semigroups of operators on Banach spaces.) Since the homomorphism $\pi_{X_{s t s g} X}$ maps the minimal idempotents of $\mathcal{U}$ onto the minimal idempotents of $\mathcal{U}_{X_{s t s g}}$, we may suppose that $X=X_{\text {stsg }}$. 
To prove (a), let $x \in X_{m}$. Then, for any minimal idempotent $d$, the set $\{u \in$ $\mathcal{U} d: u(x)=x\}$ is a nonempty, closed subsemigroup of $\mathcal{U}$ and hence contains an idempotent. Conversely, assume that $e(x)=x$ for some minimal idempotent $e \in \mathcal{U}$. If we choose $w \in \mathcal{U}$ so that weve $=e$, then $x=$ weve $(x)=$ wev $(x)$, so $x \in X_{m}$.

To prove (b) let $x \in X_{m d}$ and let $e^{2}=e \in \mathcal{U}$. Choose $u \in \mathcal{U}$ such that $u e(x)=x$. Then $e(x)=e u e(x)=u e(x)=x$, the middle equality by (c) of Example 5. The converse follows from (a) of this example and from (c) of Example 5.

As a consequence of (b), $X_{m d} \cap X_{\text {stsg }}$ is a closed, invariant subspace of $X$ and is maximal with respect to the property that $\mathcal{U}_{X_{m d} \cap X_{s t s g}}$ is a topological group with identity the identity operator. Hence, if $U_{S}$ is weakly almost periodic then $X_{m d}$ is the space $X_{\text {sap }}$ of strongly almost periodic vectors of $X[2,3]$ and $Y=X_{d}$ has the deLeeuw-Glicksberg splitting property:

$$
Y=X_{\text {sap }} \oplus(Y \cap \operatorname{ker} e) \text { for all minimal idempotents } e \in \mathcal{U} \text {. }
$$

In this connection, let $X_{\text {umri }}$ denote the subspace of $X$ which is maximal with respect to the property that $\mathcal{U}_{X_{u m r i}}$ has a unique minimal right ideal. It follows from $\left[3\right.$, Theorem 4.10] (or $[2,6.2 .14]$ ) that if $U_{S}$ is weakly almost periodic then $X_{u m r i}$ is the largest invariant subspace of $X$ with the splitting property.

We note for future use that if $U_{S}$ is weakly almost periodic and $X=X_{u m r i}$ then $X_{m d}=X_{m}$. This follows from (a) and (b) and the fact that eve $=v e$ and $d e=e$ for all minimal idempotents $d, e \in \mathcal{U}$ and all $v \in \mathcal{U}$ [2, 1.2.17].

Example 7. Call a vector $x \in X$ proximal (with respect to $U$ ) if the action of (8) is proximal, i.e., if for each pair $u, v \in \mathcal{U}$ there exists a net $\left\{U_{s_{\alpha}}\right\}$ such that $\lim _{\alpha}\left(U_{s_{\alpha}} u\right)(x)=\lim _{\alpha}\left(U_{s_{\alpha}} v\right)(x)$. Denote the set of proximal vectors by $X_{p}$. We show that if $x \in X_{\text {stsg }}$ then

$$
x \in X_{p} \Longleftrightarrow w u(x)=w(x) \text { for all } w \in K(\mathcal{U}) \text { and } u \in \mathcal{U}
$$

Note first that since $\mathcal{U}_{X_{\text {sts }}}$ is semitopological, $x \in X_{p} \Longleftrightarrow$ for each pair $u, v \in \mathcal{U}$ there exists $w \in \mathcal{U}$ such that $w u(x)=w v(x)$. Hence, if $x \in X_{p}$ then $w u(x)=w v(x)$ for all $w \in \bigcup\{\mathcal{U} e: e$ a minimal idempotent $\}=K(\mathcal{U})$. Taking $w \in \mathcal{U} e$ and $v=e$ we have $w u(x)=w e(x)=w(x)$ for all $u \in \mathcal{U}$. The converse is clear.

As a consequence, $X_{\text {stsg }} \cap X_{p}$ is a closed invariant subspace of $X$ and is maximal with respect to the property that $K\left(\mathcal{U}_{X_{s t s q} \cap X_{p}}\right)$ is the set of left zeros of $\mathcal{U}_{X_{s t s g} \cap X_{p}}$ (or, equivalently, is a left zero semigroup). Also, $X_{\text {stsg }} \cap X_{d} \cap X_{p}$ is maximal with respect to the property that $\mathcal{U}_{X_{s t s g} \cap X_{d} \cap X_{p}}$ consists of a single projection (with range $X_{s t s g} \cap X_{m d} \cap X_{p}$ ), and $X_{s t s g} \cap X_{m d} \cap X_{p}$ is maximal with respect to the property that $\mathcal{U}_{X_{s t s g} \cap X_{m d} \cap X_{p}}$ consists of the identity operator.

\section{Inductive and Projective Limits of $\mathcal{P}$-Subspaces}

In this section we prove that the property of being a $\mathcal{P}$-space is preserved under the formation of projective and strict inductive limits. We treat the easy inductive limit case first.

Theorem 3. Let $X=\bigcup_{n} X_{n}$ be the strict inductive limit of a sequence of lctvs $X_{n}$. Let $S$ be a stsg and for each $n$ let $U^{n}$ be an equicontinuous representation of $S$ 
on $X_{n}$ such that $\left.U_{s}^{n+1}\right|_{X_{n}}=U_{s}^{n}$. For $s \in S$ define $U_{s}$ on $X$ by $\left.U_{s}\right|_{X_{n}}=U_{s}^{n}$. Then $s \mapsto U_{s}$ is an equicontinuous representation of $S$ on $X$ such that

$$
X_{w c}=\bigcup_{n} X_{n w c}, \quad X_{w a p}=\bigcup_{n} X_{n w a p} \text { and } X_{a p}=\bigcup_{n} X_{n a p} .
$$

Moreover, if $\mathcal{P}$ is a regular property of compactifications of $S$ and if each $U^{n}$ is weakly continuous then $U$ is weakly continuous and

$$
X_{\mathcal{P}}=\bigcup_{n} X_{n \mathcal{P}}
$$

Proof. $U$ is clearly a representation on $X$. To see that $U$ is equicontinuous, let $p$ be a continuous seminorm on $X$. Then $\left.p\right|_{X_{n}}$ is a continuous seminorm on $X_{n}$, hence there exists a continuous seminorm $q_{n}$ on $X_{n}$ such that $p\left(U_{s} x\right) \leq q_{n}(x)$ for all $x \in X_{n}$ and $s \in S$. By definition of inductive limit topology, $q(x)=\sup _{s \in S} p\left(U_{s} x\right)$ defines a continuous seminorm on $X$ and $p\left(U_{s} x\right) \leq q(x)$ for all $x \in X$ and $s \in S$.

The assertions regarding the spaces $X_{w c}, X_{w a p}$, and $X_{a p}$ are clear, since the original topology of $X_{n}$ is the relative topology from $X$. The last assertion of the theorem follows from the observation that $X_{n \mathcal{P}}=X_{\mathcal{P}} \cap X_{n}$.

Corollary. If, for each $n, X_{n}$ is a strictly convex Banach space, $U^{n}$ is weakly almost periodic and $\left\|U_{s}^{n}\right\| \leq 1$ for all $s$, then $X$ has the splitting property (see Example 6).

Proof. The strict convexity implies that $X_{n}=X_{n \text { umri }}$ [4, Corollary 4.12] or [2, 6.2.15]. Hence, by Theorem $3, X=X_{u m r i}$, so $X$ has the splitting property.

Theorem 3 may be applied to the following typical setting: Let $S$ be a topological group acting continuously on the right on a locally compact topological space $\Omega$ and let $\left\{\Omega_{n}\right\}$ be an increasing sequence of open invariant sets whose union is $\Omega$. Let $X_{n}$ be the normed linear space (sup norm) of all continuous, complex valued functions on $\Omega$ with compact support contained in $\Omega_{n}$ and define $U_{s}^{n}$ on $X_{n}$ by $U_{s}^{n} f(\omega)=f(\omega s)$. The inductive limit of the spaces $X_{n}$ is the space of all continuous functions on $\Omega$ with compact support.

For the projective limit case we need the following lemma.

Lemma 3. Let $U$ and $V$ be weakly continuous, equicontinuous representations of stsgs $S$ and $T$ on lctvs $X$ and $Y$, respectively. If $\theta: S \mapsto T$ is a continuous, surjective homomorphism and $w \in \mathcal{L}(X, Y)$ satisfies $w \circ U_{s}=V_{\theta(s)} \circ w$ for all $s \in S$, then there exists a continuous, surjective semigroup homomorphism $\pi_{X}^{w}$ : $\mathcal{U} \mapsto \mathcal{V}_{w(X)}$ such that $\pi_{X}^{w}(u) \circ w=w^{\prime \prime} \circ u$ and $\pi_{X}^{w}\left(U_{s}\right)=\left.V_{\theta(s)}\right|_{w(X)}$. Moreover, if $T=S$ and if $\theta$ is the identity map on $S$ then $\pi_{Z}^{w} \circ \pi_{Z X}=\pi_{X}^{w}$ for any $U$-invariant subspace $Z$ of $X$ with $w(Z)=Y$.

Proof. We may assume that $w$ is surjective. For $u \in \mathcal{U}$ define $\pi_{X}^{w}(u) \in \mathcal{L}(Y)$ by

$$
\begin{gathered}
\qquad \pi_{X}^{w}(u)(w(x))=w^{\prime \prime}(u(x)) \quad(x \in X) . \\
\text { If } U_{s_{\alpha}} \stackrel{w^{*}}{\rightarrow} u \text { and } V_{\theta\left(s_{\alpha}\right)} \stackrel{w^{*}}{\rightarrow} v \text { then } \\
\left\langle y^{\prime}, w^{\prime \prime}(u(x))\right\rangle=\lim _{\alpha}\left\langle U_{s_{\alpha}}(x), w^{\prime}\left(y^{\prime}\right)\right\rangle=\lim _{\alpha}\left\langle V_{\theta\left(s_{\alpha}\right)}(w(x)), y^{\prime}\right\rangle=\left\langle y^{\prime}, v(w(x))\right\rangle,
\end{gathered}
$$


hence $\pi_{X}^{w}(u)$ is well defined and equals $v$. Therefore $\pi_{X}^{w}$ maps $\mathcal{U}$ into $\mathcal{V}$ such that $\pi_{X}^{w}\left(U_{s}\right)=V_{\theta(s)}$. Since $\pi_{X}^{w}(u)$ is obviously continuous, it is a semigroup homomorphism.

To prove the last assertion of the lemma, let $u \in \mathcal{U}, x \in X$ and $y^{\prime} \in Y^{\prime}$. Then

$$
\begin{aligned}
\left\langle y^{\prime}, \pi_{Z}^{w} \circ \pi_{Z X}(u)(w(x))\right\rangle & =\left\langle\left(\left.w\right|_{Z}\right)^{\prime}\left(y^{\prime}\right), \pi_{z X}(u)(x)\right\rangle=\left\langle\left. w^{\prime}\left(y^{\prime}\right)\right|_{Z}, \pi_{z X}(u)(x)\right\rangle \\
& =\left\langle w^{\prime}\left(y^{\prime}\right), u(x)\right\rangle=\left\langle y^{\prime}, \pi_{X}^{w}(u)(w(x))\right\rangle .
\end{aligned}
$$

\section{Corollary.}

(a) If $\mathcal{P}$ is a regular property of compact left topological semigroups then $w\left(X_{\mathcal{P}}\right) \subset$ $Y_{\mathcal{P}}$.

(b) If $\mathcal{P}$ is a regular property of compactifications of $S$ and if $T=S$ and $\theta$ is the identity map then $w\left(X_{\mathcal{P}}\right) \subset Y_{\mathcal{P}}$.

For the next result recall that if $X$ is the direct product $\prod_{i \in I} X_{i}$ of a family of lctvs $X_{i}$ then $X_{\beta}^{\prime}$ may be identified with the locally convex direct sum $\bigoplus_{i \in I} X_{i \beta}^{\prime}$ under $x^{\prime} \leftrightarrow \sum_{i} x_{i}^{\prime}$, where $x^{\prime}(x)=\sum_{i}\left\langle x_{i}, x_{i}^{\prime}\right\rangle, x=\left(x_{i}\right)_{i} \in X$ [11, p. 192]. Thus $X^{\prime \prime}=$ $\left(X_{\beta}^{\prime}\right)^{\prime}$ is algebraically isomorphic to $\prod_{i \in I} X_{i}^{\prime \prime}$ under $x^{\prime \prime} \leftrightarrow\left(x_{i}^{\prime \prime}\right)_{i}$, where $x^{\prime \prime}\left(\sum_{i} x_{i}^{\prime}\right)=$ $\sum_{i}\left\langle x_{i}^{\prime}, x_{i}^{\prime \prime}\right\rangle\left[11\right.$, p. 138]. Since the natural topology of $X^{\prime \prime}$ is the topology of uniform convergence on subsets of $\bigoplus_{i \in I} X_{i}^{\prime}$ of the form $\left(\prod_{i \in a} N_{i} \times \prod_{i \notin a} X_{i}\right)^{\circ}=\Gamma_{i \in a} N_{i}^{\circ}$, where $N_{i}$ is a convex, circled neighborhood of 0 in $X_{i}, a$ is a finite subset of $I$, and $\Gamma$ denotes the convex circled hull operation, it follows that the natural topology of $X^{\prime \prime}$ is the product of the natural topologies of the $X_{i}^{\prime \prime}$.

Theorem 4. For each $i \in I$ let $U^{i}$ be an equicontinuous representation of a stsg $S_{i}$ on a lctvs $X_{i}$. Define the product representation $U=\prod_{i \in I} U^{i}$ of $T:=\prod_{i \in I} S_{i}$ on $X:=\prod_{i \in I} X_{i}$ by

$$
U_{s}(x)=\left(U_{s_{i}}^{i} x_{i}\right)_{i} \quad\left(s=\left(s_{i}\right)_{i} \in T, x=\left(x_{i}\right)_{i} \in X\right) .
$$

\section{Then}

(a) $U$ is equicontinuous and $\mathcal{U}=\prod_{i} \mathcal{U}^{i}$;

(b) $X_{w c}=\prod_{i \in I} X_{i w c}, X_{w a p}=\prod_{i \in I} X_{i w a p}$ and $X_{a p}=\prod_{i \in I} X_{i a p}$;

(c) if each $U^{i}$ is weakly continuous and if $\mathcal{P}$ is a regular property of compact left topological semigroups which is inherited by direct products then $X_{\mathcal{P}}=$ $\prod_{i \in I} X_{i \mathcal{P}}$

Proof. Part (a) is clear, in light of the above discussion. The first equality in (b) follows from $\sigma\left(X, X^{\prime}\right)=\prod_{i} \sigma\left(X_{i}, X_{i}^{\prime}\right)$, and the remaining equalities follow from $U_{T} x=\prod_{i} U_{S_{i}}^{i} x_{i}$. To prove (c), let $Y_{i}:=X_{i \mathcal{P}}$ and $Y:=\prod_{i \in I} Y_{i}$ and note that since $\mathcal{U}_{Y}=\prod_{i} \mathcal{U}_{Y_{i}}^{i}$ and $\mathcal{P}$ is inherited by direct products, $Y \subset X_{\mathcal{P}}$. For the reverse inclusion apply the corollary of Lemma 3 to the projection transformation $w_{j}: \prod_{i \in I} X_{i} \mapsto X_{j}$ and the projection homomorphism $\theta_{j}: T \mapsto S_{j}$ to obtain $w_{j}\left(X_{\mathcal{P}}\right) \subset Y_{j}$.

Theorem 5. Let $I$ be a directed set and for each $i \in I$ let $U^{i}$ be an equicontinuous representation of a stsg $S$ on a lctvs $X_{i}$. Let

$$
Y:=\lim _{\longleftarrow} X_{i}=\left\{\left(x_{i}\right)_{i} \in \prod_{i \in I} X_{i}: w_{i j}\left(x_{j}\right)=x_{i}, i \leq j\right\}
$$


be a projective limit of the spaces $X_{i}$, where the $w_{i j} \in \mathcal{L}\left(X_{j}, X_{i}\right)$ satisfy $w_{i i}=\mathrm{id}_{X_{i}}$, $w_{i j} \circ w_{j k}=w_{i k}$, and $w_{i j} \circ U_{s}^{j}=U_{s}^{i} \circ w_{i j}, i \leq j \leq k$. Define $V_{s}$ on $Y$ by $V_{s}\left(\left(x_{i}\right)_{i}\right)=$ $\left(U_{s}^{i} x_{i}\right)_{i}$. Then $V$ is an equicontinuous representation of $S$ and

$$
Y_{w c}=\lim _{\longleftarrow} X_{i w c}, \quad Y_{w a p}=\lim _{\longleftarrow} X_{i w a p}, \quad \text { and } \quad Y_{a p}=\lim _{\longleftarrow} X_{i a p}
$$

(relative to the appropriate restrictions of the mappings $w_{i j}$ ). If each $U^{i}$ is weakly continuous and if $\mathcal{P}$ is a regular property of compact left topological semigroups inherited by direct products, then

$$
Y_{\mathcal{P}}=\lim _{\longleftarrow} X_{i \mathcal{P}}
$$

Proof. The projective limits in the statement of the theorem exist since $w_{i j}\left(X_{j w c}\right) \subset$ $X_{i w c}, w_{i j}\left(X_{j w a p}\right) \subset X_{i w a p}, w_{i j}\left(X_{j a p}\right) \subset X_{i a p}$ and, by the corollary to Lemma $3, w_{i j}\left(X_{j \mathcal{P}}\right) \subset X_{i \mathcal{P}}$. Let $U, X$ and $T$ be as in the statement of Theorem 4 . The representation $V$ in the statement of the present theorem is the restriction to $Y$ of the representation $s \mapsto W_{s}:=U_{\iota(s)}$ on $X$, where $\iota: S \mapsto T$ denotes the injection onto the diagonal. Clearly, $W$ is equicontinuous and has properties (b) and (c) of Theorem 4. The theorem now follows from the observation that $Y \cap X_{\mathcal{P}}=Y_{P}$, $Y \cap X_{w c}=Y_{w c}$, etc.

Here's a simple example to which Theorem 5 may be applied: Let $\left\{t_{i}: i \in I\right\}$ be a net of units of a stsg $S$ with identity and set $s_{i j}=t_{j}^{-1} t_{i}, i \leq j$. Let $F$ be a translation invariant, linear subspace of $C(S)$ and let $U_{s}^{i}=R_{s}$ on $X_{i}=F$. Then the left translation operators $w_{i j}=L_{s_{i j}}$ have the required properties.

For another example, let $\mathcal{J}$ be a family of right ideals of a stsg $S$ such that $\cup \mathcal{J}=S$ and let $C(S, \mathcal{J})$ denote the set of all continuous, complex-valued functions on $S$ which are bounded on each member of $\mathcal{J}$. The right translation representation of $S$ on $C(S, \mathcal{J})$ is obviously equicontinuous with respect to the locally convex topology generated by the seminorms $p_{J}: f \mapsto \sup _{s \in J}|f(s)|, J \in \mathcal{J}$, and is a uniformly bounded representation on the Banach space $C(J)$. If $\mathcal{J}$ is directed upward by inclusion then $C(S, \mathcal{J})$ is a projective limit of the Banach spaces $C(J)$ relative to the restriction mappings $w_{J K}: C(K) \mapsto C(J), J, K \in \mathcal{J}, J \subset K$ (which clearly commute with $\left.R_{s}, s \in S\right)$. For example, one could take $S=(0,+\infty)$ under addition or $\vee$ and $\mathcal{J}=\{(1 / n,+\infty): n \in \mathbb{N}\}$.

\section{Tensor Products of $\mathcal{P}$-Subspaces}

If $X$ and $Y$ are lctvs we denote by $X \otimes Y$ the completion of the algebraic tensor product $X \odot Y$ in the topology of bi-equicontinuous convergence. This is the topology generated by the seminorms

$$
p_{A^{\prime} \otimes B^{\prime}}(z):=\sup \left\{\left|\left\langle z, z^{\prime}\right\rangle\right|: z^{\prime} \in A^{\prime} \otimes B^{\prime}\right\}
$$

where $A^{\prime} \subset X^{\prime}$ and $B^{\prime} \subset Y^{\prime}$ are equicontinuous, $A^{\prime} \otimes B^{\prime}:=\left\{x^{\prime} \otimes y^{\prime}: x^{\prime} \in A^{\prime}, y^{\prime} \in\right.$ $\left.B^{\prime}\right\}$ and $x^{\prime} \otimes y^{\prime}$ is the unique member of $(X \otimes Y)^{\prime}$ satisfying $\left\langle x \otimes y, x^{\prime} \otimes y^{\prime}\right\rangle=$ $\left\langle x, x^{\prime}\right\rangle\left\langle y, y^{\prime}\right\rangle$. More generally, if $X_{1}, X_{2}, \ldots, X_{n}$ are lctvs then $X_{1} \otimes X_{2} \otimes \cdots \otimes X_{n}$ denotes the completion of the algebraic tensor product $X_{1} \odot X_{2} \odot \cdots \odot X_{n}$ in the topology of $n$-fold-equicontinuous convergence. Equivalently, $X_{1} \otimes X_{2} \otimes \cdots \otimes X_{n}$ may be defined inductively as $\left(X_{1} \otimes X_{2} \otimes \cdots \otimes X_{n-1}\right) \otimes X_{n}$.

In this section we determine general conditions under which the tensor product of $\mathcal{P}$-spaces is a $\mathcal{P}$-space. We begin with the following key result. 
Lemma 4. Let $X$ and $Y$ be lctvs. Then $(x, y) \mapsto x \otimes y: X \times Y \mapsto X \otimes Y$ extends to a continuous bilinear mapping $\left(x^{\prime \prime}, y^{\prime \prime}\right) \mapsto x^{\prime \prime} \otimes y^{\prime \prime}: X^{\prime \prime} \times Y^{\prime \prime} \mapsto(X \otimes Y)^{\prime \prime}$ such that

(a) $x^{\prime \prime} \mapsto x^{\prime \prime} \otimes y^{\prime \prime}: X^{\prime \prime} \mapsto(X \otimes Y)^{\prime \prime}$ is weak ${ }^{*}$ continuous for each $y^{\prime \prime} \in Y^{\prime \prime}$;

(b) $y^{\prime \prime} \mapsto x \otimes y^{\prime \prime}: Y^{\prime \prime} \mapsto(X \otimes Y)^{\prime \prime}$ is weak ${ }^{*}$ continuous for each $x \in X$;

(c) $\left\langle x^{\prime} \otimes y^{\prime}, x^{\prime \prime} \otimes y^{\prime \prime}\right\rangle=\left\langle x^{\prime}, x^{\prime \prime}\right\rangle\left\langle y^{\prime}, y^{\prime \prime}\right\rangle$ for all $x^{\prime} \in X^{\prime}$ and $y^{\prime} \in Y^{\prime}$;

(d) if $X$ and $Y$ are normed spaces then $\left\|x^{\prime \prime} \otimes y^{\prime \prime}\right\| \leq\left\|x^{\prime \prime}\right\|\left\|y^{\prime \prime}\right\|$.

Proof. For $x \in X$ define $\ell_{x} \in \mathcal{L}(Y, X \otimes Y)$ by $\ell_{x}(y)=x \otimes y$ and set $x \otimes y^{\prime \prime}:=\ell_{x}^{\prime \prime}\left(y^{\prime \prime}\right)$. The mapping $\left(x, y^{\prime \prime}\right) \mapsto x \otimes y^{\prime \prime}: X \times Y^{\prime \prime} \rightarrow(X \otimes Y)^{\prime \prime}$ is clearly bilinear, and for $z^{\prime} \in(X \otimes Y)^{\prime}$ and $y \in Y$ we have $\left\langle x \otimes y, z^{\prime}\right\rangle=\left\langle y, \ell_{x}^{\prime}\left(z^{\prime}\right)\right\rangle=\left\langle z^{\prime}, \ell_{x}^{\prime \prime}(\hat{y})\right\rangle=\left\langle z^{\prime}, x \otimes \hat{y}\right\rangle$, so $y^{\prime \prime} \mapsto x \otimes y^{\prime \prime}$ is an extension of $y \mapsto x \otimes y$. Moreover, (b) holds since $\left\langle z^{\prime}, x \otimes y^{\prime \prime}\right\rangle=$ $\left\langle\ell_{x}^{\prime}\left(z^{\prime}\right), y^{\prime \prime}\right\rangle$.

Let $C^{\prime}$ be an equicontinuous subset of $(X \otimes Y)^{\prime}$ and choose equicontinuous sets $A^{\prime} \subset X^{\prime}$ and $B^{\prime} \subset Y^{\prime}$ such that $p_{C^{\prime}} \leq p_{A^{\prime} \otimes B^{\prime}}$. Then

$$
\left|\left\langle y, \ell_{x}^{\prime}\left(z^{\prime}\right)\right\rangle\right|=\left|\left\langle x \otimes y, z^{\prime}\right\rangle\right| \leq p_{A^{\prime}}(x) p_{B^{\prime}}(y) \quad\left(x \in X, y \in Y, z^{\prime} \in C^{\prime}\right) ;
$$

hence $B_{1}^{\prime}:=\left\{\ell_{x}^{\prime}\left(z^{\prime}\right): p_{A^{\prime}}(x) \leq 1, z^{\prime} \in C^{\prime}\right\}$ is an equicontinuous subset of $Y^{\prime}$ and $q_{C^{\prime}}\left(x \otimes y^{\prime \prime}\right) \leq q_{B_{1}^{\prime}}\left(y^{\prime \prime}\right)$ for all $x$ with $p_{A^{\prime}}(x) \leq 1$. Therefore

$$
q_{C^{\prime}}\left(x \otimes y^{\prime \prime}\right) \leq p_{A^{\prime}}(x) q_{B_{1}^{\prime}}\left(y^{\prime \prime}\right) \quad\left(x \in X, y^{\prime \prime} \in Y^{\prime \prime}\right)
$$

Fix $y^{\prime \prime} \in Y^{\prime \prime}$ and define $r_{y^{\prime \prime}}:(X \otimes Y)^{\prime} \mapsto X^{\prime}$ by

$$
\left\langle x, r_{y^{\prime \prime}}\left(z^{\prime}\right)\right\rangle=\left\langle z^{\prime}, x \otimes y^{\prime \prime}\right\rangle \quad\left(x \in X, z^{\prime} \in(X \otimes Y)^{\prime}\right) .
$$

Let $A$ be any bounded subset of $X$ and let $B=\left\{y_{\alpha}\right\}$ be a bounded net in $Y$ such that $y_{\alpha} \stackrel{w^{*}}{\rightarrow} y^{\prime \prime}$. Then $A \otimes B:=\{x \otimes y: x \in A, y \in B\}$ is bounded in $X \otimes Y$ and

$\left|\left\langle x, r_{y^{\prime \prime}}\left(z^{\prime}\right)\right\rangle\right|=\lim _{\alpha}\left|\left\langle x \otimes y_{\alpha}, z^{\prime}\right\rangle\right| \leq \sup \left\{\left|\left\langle z, z^{\prime}\right\rangle\right|: z \in A \otimes B\right\} \quad\left(x \in A, z^{\prime} \in(X \otimes Y)^{\prime}\right)$.

Thus $r_{y^{\prime \prime}} \in \mathcal{L}\left((X \otimes Y)_{\beta}^{\prime}, X_{\beta}^{\prime}\right)$ so $r_{y^{\prime \prime}}^{\prime}: X^{\prime \prime} \mapsto(X \otimes Y)^{\prime \prime}$. Set $x^{\prime \prime} \otimes y^{\prime \prime}:=r_{y^{\prime \prime}}^{\prime}\left(x^{\prime \prime}\right)$. Then $\left(x^{\prime \prime}, y^{\prime \prime}\right) \mapsto x^{\prime \prime} \otimes y^{\prime \prime}$ is bilinear and for any $z^{\prime} \in(X \otimes Y)^{\prime}$ and $x \in X$ we have $\left\langle z^{\prime}, \hat{x} \otimes y^{\prime \prime}\right\rangle=\left\langle z^{\prime}, r_{y^{\prime \prime}}^{\prime}(\hat{x})\right\rangle=\left\langle x, r_{y^{\prime \prime}}\left(z^{\prime}\right)\right\rangle=\left\langle z^{\prime}, x \otimes y^{\prime \prime}\right\rangle$, so $x^{\prime \prime} \mapsto x^{\prime \prime} \otimes y^{\prime \prime}$ is an extension of $x \mapsto x \otimes y^{\prime \prime}$. Moreover, (a) holds since $\left\langle z^{\prime}, x^{\prime \prime} \otimes y^{\prime \prime}\right\rangle=\left\langle r_{y^{\prime \prime}}^{\prime}\left(z^{\prime}\right), x^{\prime \prime}\right\rangle$.

To establish the continuity of $\left(x^{\prime \prime}, y^{\prime \prime}\right) \mapsto x^{\prime \prime} \otimes y^{\prime \prime}$, let $C^{\prime}$ be an equicontinuous subset of $(X \otimes Y)^{\prime}$ and choose equicontinuous sets $A^{\prime} \subset X^{\prime}$ and $B_{1}^{\prime} \subset Y^{\prime}$ as in (9). Then

$$
\left|\left\langle x, r_{y^{\prime \prime}}\left(z^{\prime}\right)\right\rangle\right|=\left|\left\langle z^{\prime}, x \otimes y^{\prime \prime}\right\rangle\right| \leq p_{A^{\prime}}(x) q_{B_{1}^{\prime}}\left(y^{\prime \prime}\right) \quad\left(x \in X, y^{\prime \prime} \in Y^{\prime \prime}, z^{\prime} \in C^{\prime}\right),
$$

hence $A_{1}^{\prime}:=\left\{r_{y^{\prime \prime}}\left(z^{\prime}\right): q_{B_{1}^{\prime}}\left(y^{\prime \prime}\right) \leq 1, z^{\prime} \in C^{\prime}\right\}$ is an equicontinuous subset of $X^{\prime}$ and $q_{C^{\prime}}\left(x^{\prime \prime} \otimes y^{\prime \prime}\right) \leq q_{A_{1}^{\prime}}\left(x^{\prime \prime}\right)$ for all $y^{\prime \prime}$ with $q_{B_{1}^{\prime}}\left(y^{\prime \prime}\right) \leq 1$. Thus

$$
q_{C^{\prime}}\left(x^{\prime \prime} \otimes y^{\prime \prime}\right) \leq q_{A_{1}^{\prime}}\left(x^{\prime \prime}\right) q_{B_{1}^{\prime}}\left(y^{\prime \prime}\right) \quad\left(x^{\prime \prime} \in X^{\prime \prime}, y^{\prime \prime} \in Y^{\prime \prime}\right),
$$

which shows that $\left(x^{\prime \prime}, y^{\prime \prime}\right) \mapsto x^{\prime \prime} \otimes y^{\prime \prime}$ is continuous. 
Part (c) is a consequence of (a) and (b) and the weak* density of a locally convex space in its bidual. Part (d) follows easily from (10).

Note that the bilinear extension $\left(x^{\prime \prime}, y^{\prime \prime}\right) \mapsto x^{\prime \prime} \otimes y^{\prime \prime}$ of $(x, y) \mapsto x \otimes y$ is unique with respect to properties (a) and (b) of Lemma 4. We shall refer to this extension as the right continuous tensor product. Obviously, a unique left continuous tensor product also exists. For finitely many lctvs $X_{1}, X_{2}, \ldots, X_{n}$ we define the (right continuous) tensor product $x_{1}^{\prime \prime} \otimes x_{2}^{\prime \prime} \otimes \cdots \otimes x_{n}^{\prime \prime}$ inductively by

$$
x_{1}^{\prime \prime} \otimes x_{2}^{\prime \prime} \otimes \cdots \otimes x_{n}^{\prime \prime}=\left(x_{1}^{\prime \prime} \otimes x_{2}^{\prime \prime} \otimes \cdots \otimes x_{n-1}^{\prime \prime}\right) \otimes x_{n}^{\prime \prime} .
$$

Lemma 5. Let $\mathcal{S}$ and $\mathcal{T}$ be equicontinuous semigroups of operators on the lctvs $X$ and $Y$, respectively, and let $\mathcal{S} \otimes \mathcal{T}:=\{u \otimes v: u \in \mathcal{S}, v \in \mathcal{T}\}$. For each $u \in \overline{\mathcal{S}}$ and $v \in \overline{\mathcal{T}}$ there exists a unique member $u \otimes v$ of $\overline{\mathcal{S} \otimes \mathcal{T}}$ (weak ${ }^{*}$ operator closures) such that $(u \otimes v)(x \otimes y)=u(x) \otimes v(y)$ (= the right continuous tensor product of $u(x)$ and $v(y))$. Moreover,

$$
(u \otimes v)\left(u_{1} \otimes v_{1}\right)=u u_{1} \otimes v v_{1} \quad\left(u \in \overline{\mathcal{S}} \cap \mathcal{L}(X), u_{1} \in \overline{\mathcal{S}}, v, v_{1} \in \overline{\mathcal{T}}\right) .
$$

Proof. Let $\left\{u_{\alpha}\right\} \subset \mathcal{S}$ and $\left\{v_{\beta}\right\} \subset \mathcal{T}$ be nets such that $u_{\alpha} \stackrel{w^{*} o p}{\rightarrow} u$ and $v_{\beta} \stackrel{w^{*} o p}{\rightarrow} v$. Since the mapping $(x, y) \mapsto u(x) \otimes v(y): X \times Y \mapsto(X \otimes Y)^{\prime \prime}$ is bilinear it factors through a linear mapping $u \otimes v: X \odot Y \mapsto(X \otimes Y)^{\prime \prime}$. If $z=\sum_{i} x_{i} \otimes y_{i} \in X \odot Y$ and $z^{\prime} \in(X \otimes Y)^{\prime}$ then from (a) and (b) of Lemma 4

$$
\begin{aligned}
\left\langle z^{\prime},(u \otimes v)(z)\right\rangle & =\left\langle z^{\prime}, \sum_{i} u\left(x_{i}\right) \otimes v\left(y_{i}\right)\right\rangle \\
& =\lim _{\alpha} \lim _{\beta}\left\langle z^{\prime}, \sum_{i} u_{\alpha}\left(x_{i}\right) \otimes v_{\beta}\left(y_{i}\right)\right\rangle \\
& =\lim _{\alpha} \lim _{\beta}\left\langle\left(u_{\alpha} \otimes v_{\beta}\right)(z), z^{\prime}\right\rangle .
\end{aligned}
$$

Let $C^{\prime}$ be an equicontinuous subset of $(X \otimes Y)^{\prime}$ and choose equicontinuous subsets $A^{\prime} \subset X^{\prime}$ and $B^{\prime} \subset Y^{\prime}$ such that $p_{C^{\prime}} \leq p_{A^{\prime} \otimes B^{\prime}}$. Then $A_{1}{ }^{\prime}:=\mathcal{S}^{\prime}\left(A^{\prime}\right)$ and $B_{1}{ }^{\prime}:=$ $\mathcal{T}^{\prime}\left(B^{\prime}\right)$ are equicontinuous and $\left|\left\langle\left(u_{\alpha} \otimes v_{\beta}\right)(z), z^{\prime}\right\rangle\right| \leq p_{A_{1}{ }^{\prime} \otimes B_{1}{ }^{\prime}}(z)$ for all $\alpha, \beta$ and all $z^{\prime} \in C^{\prime}$. From $(12), q_{C^{\prime}}((u \otimes v)(z)) \leq p_{A_{1^{\prime}} \otimes B_{1^{\prime}}}(z)$ for all $z \in X \odot Y$ and all $u \in \overline{\mathcal{S}}, v \in \overline{\mathcal{T}}$. Thus $u \otimes v$ is continuous on $X \odot Y$ and hence may be extended continously to $X \otimes Y$. By (12) and the equicontinuity of $\mathcal{S} \otimes \mathcal{T},(u \otimes v)(z)=$ $w^{*}-\lim _{\alpha} \lim _{\beta}\left(u_{\alpha} \otimes v_{\beta}\right)(z)$ for $z \in X \otimes Y$ so $u \otimes v=\lim _{\alpha} \lim _{\beta} u_{\alpha} \otimes v_{\beta} \in \overline{\mathcal{S} \otimes \mathcal{T}}$.

To verify $(11)$, let $U_{s_{\alpha}} \stackrel{w^{*} o p}{\rightarrow} u_{1}$ and $V_{t_{\beta}} \stackrel{w^{*} o p}{\rightarrow} v_{1}$. Then in the weak* operator topology

$$
\begin{aligned}
(u \otimes v)\left(u_{1} \otimes v_{1}\right) & =\lim _{\alpha} \lim _{\beta}(u \otimes v)\left(U_{s_{\alpha}} \otimes V_{t_{\beta}}\right) \\
& =\lim _{\alpha} \lim _{\beta}\left(u U_{s_{\alpha}}\right) \otimes\left(v V_{t_{\beta}}\right) \\
& =u u_{1} \otimes v v_{1}
\end{aligned}
$$

the last equality because $u U_{s_{\alpha}} \in \mathcal{L}(X)$. 
Lemma 6. Let $A$ and $B$ be relatively weakly compact subsets of $X$ and $Y$, respectively. Then $A \otimes B:=\{x \otimes y: x \in A, y \in B\}$ is relatively weakly compact.

Proof. We use the double limit criterion of Grothendieck [11, p. 187]. Let $\left\{x_{n} \otimes y_{n}\right\}$ be a sequence in $A \otimes B$ and let $\left\{z_{n}^{\prime}\right\}$ be an equicontinuous sequence in $(X \otimes Y)^{\prime}$ such that the limits $a:=\lim _{m} \lim _{n}\left\langle x_{n} \otimes y_{n}, z_{m}^{\prime}\right\rangle$ and $b:=\lim _{n} \lim _{m}\left\langle x_{n} \otimes y_{n}, z_{m}^{\prime}\right\rangle$ exist. Let $x_{0}$ and $y_{0}$ be weak cluster points of $\left\{x_{n}\right\}$ and $\left\{y_{n}\right\}$, respectively, and let $z_{0}^{\prime}$ be a weak* cluster point of $\left\{z_{n}^{\prime}\right\}$ (Alaoglu's Theorem). There exist weak* closed, equicontinuous subsets $A^{\prime}$ of $X^{\prime}$ and $B^{\prime}$ of $Y^{\prime}$ and a bounded sequence of complex Borel measures $\mu_{m}$ on $A^{\prime} \times B^{\prime}$ (where the latter has the product of the relative weak* topologies) such that

$$
\left\langle x \otimes y, z_{m}^{\prime}\right\rangle=\int_{A^{\prime} \times B^{\prime}}\left\langle x, x^{\prime}\right\rangle\left\langle y, y^{\prime}\right\rangle d \mu_{m}\left(x^{\prime}, y^{\prime}\right) \quad(x \in X, y \in Y, m \geq 0)
$$

$[11,9.2]$. Choose subsequences $\left\{x_{n_{k}}\right\}$ and $\left\{y_{n_{k}}\right\}$ such that $\left\langle x_{n_{k}}, x^{\prime}\right\rangle \rightarrow\left\langle x_{0}, x^{\prime}\right\rangle$ for all $x^{\prime} \in A^{\prime}$ and $\left\langle y_{n_{k}}, y^{\prime}\right\rangle \rightarrow\left\langle y_{0}, y^{\prime}\right\rangle$ for all $y^{\prime} \in B^{\prime}$. This is possible by the weak* compactness of $A^{\prime}$ and $B^{\prime}$ (see [11, p. 186]). By Lebesgue's dominated convergence theorem,

$$
\begin{aligned}
a & =\lim _{m} \lim _{n} \int_{A^{\prime} \times B^{\prime}}\left\langle x_{n}, x^{\prime}\right\rangle\left\langle y_{n}, y^{\prime}\right\rangle d \mu_{m}\left(x^{\prime}, y^{\prime}\right) \\
& =\lim _{m} \int_{A^{\prime} \times B^{\prime}}\left\langle x_{0}, x^{\prime}\right\rangle\left\langle y_{0}, y^{\prime}\right\rangle d \mu_{m}\left(x^{\prime}, y^{\prime}\right) \\
& =\lim _{m}\left\langle x_{0} \otimes y_{0}, z_{m}^{\prime}\right\rangle=\left\langle x_{0} \otimes y_{0}, z_{0}^{\prime}\right\rangle \\
& =\lim _{n} \int_{A^{\prime} \times B^{\prime}}\left\langle x_{n}, x^{\prime}\right\rangle\left\langle y_{n}, y^{\prime}\right\rangle d \mu_{0}\left(x^{\prime}, y^{\prime}\right) \\
& =\lim _{n}\left\langle x_{n} \otimes y_{n}, z_{0}^{\prime}\right\rangle \\
& =b
\end{aligned}
$$

as required.

The following theorem generalizes Theorem 2.2 of [7].

Theorem 6. Let $U$ and $V$ be weakly continuous, equicontinuous representations of stsgs $S$ and $T$ on lctvs $X$ and $Y$, respectively. Suppose that one of the following holds:

(a) $U$ and $V$ are weakly almost periodic;

(b) $Y_{\beta}^{\prime}$ is separable;

(c) $V$ is almost periodic.

Then

(i) $W_{(s, t)}:=U_{s} \otimes V_{t}$ defines a weakly continuous, equicontinuous representation $W=U \otimes V$ of $S \times T$ on $X \otimes Y$ such that $\mathcal{W}=\mathcal{U} \otimes \mathcal{V}$;

(ii) $K(\mathcal{W})=K(\mathcal{U}) \otimes K(\mathcal{V})$;

(iii) if $U$ and $V$ are (weakly) almost periodic then $W$ is (weakly) almost periodic;

(iv) if $\mathcal{P}$ and $\mathcal{Q}$ are regular properties of compactifications of $S$ and $T$, respectively, and if $U$ is a $\mathcal{P}$-representation and $V$ is a $\mathcal{Q}$-representation then the compactification $\mathcal{U} \otimes \mathcal{V}$ of $S \times T$ is a factor of the direct product compactification $S^{\mathcal{P}} \times T^{\mathcal{Q}}$. 
Proof. We claim that under condition (a), (b), or (c) the mapping $(u, v) \mapsto u \otimes v$ : $\mathcal{U} \times \mathcal{V} \mapsto \mathcal{U} \otimes \mathcal{V}$ is jointly continuous in the weak* operator topologies. Assuming this for the moment, we see immediately that (i) and (iii) hold, the latter by Lemma 6. Moreover, if $\theta_{S}: S^{\mathcal{P}} \mapsto \mathcal{U}$ and $\theta_{T}: T^{\mathcal{Q}} \mapsto \mathcal{V}$ are the compactification homomorphisms in (iv) then $\left(s^{\prime}, t^{\prime}\right) \mapsto \theta_{S}\left(s^{\prime}\right) \otimes \theta_{T}\left(t^{\prime}\right): S^{\mathcal{P}} \times T^{\mathcal{Q}} \mapsto \mathcal{U} \otimes \mathcal{V}$ is continuous in the weak* operator topology and hence is a compactification homomorphism.

To prove the claim, let $u_{\alpha} \rightarrow u$ in $\mathcal{U}$ and $v_{\alpha} \rightarrow v$ in $\mathcal{V}$. Suppose that (a) holds. Then $\mathcal{W}=\mathcal{U} \otimes \mathcal{V} \subset \mathcal{L}(X \otimes Y)$. We show that $u \otimes v$ is the unique limit point of the net $\left\{u_{\alpha} \otimes v_{\alpha}\right\}$. Let a subnet $\left\{u_{\beta} \otimes v_{\beta}\right\}$ converge in the weak operator topology to some $w \in \mathcal{W}$. For $x \in X, y \in Y, x^{\prime} \in X^{\prime}$ and $y^{\prime} \in Y^{\prime}$ we have

$$
\begin{aligned}
\left\langle w(x \otimes y), x^{\prime} \otimes y^{\prime}\right\rangle & =\lim _{\beta}\left\langle u_{\beta}(x) \otimes v_{\beta}(y), x^{\prime} \otimes y^{\prime}\right\rangle \\
& =\lim _{\beta}\left\langle u_{\beta}(x), x^{\prime}\right\rangle\left\langle v_{\beta}(y), y^{\prime}\right\rangle \\
& =\left\langle u(x), x^{\prime}\right\rangle\left\langle v(y), y^{\prime}\right\rangle \\
& =\left\langle u(x) \otimes v(y), x^{\prime} \otimes y^{\prime}\right\rangle .
\end{aligned}
$$

Therefore $w=u \otimes v$, which proves the claim in case (a).

For the remaining cases note that by virtue of (a) of Lemma 4 and the identity

$$
u_{\alpha}(x) \otimes v_{\alpha}(y)-u(x) \otimes v(y)=\left(u_{\alpha}(x)-u(x)\right) \otimes v(y)+u_{\alpha}(x) \otimes\left(v_{\alpha}(y)-v(y)\right)
$$

it suffices to show that for $z^{\prime} \in(X \otimes Y), c_{\alpha}=c_{\alpha}\left(x, y, z^{\prime}\right):=\left\langle z^{\prime}, u_{\alpha}(x) \otimes\left(v_{\alpha}(y)-\right.\right.$ $v(y))\rangle \rightarrow 0$. This is obvious if (c) holds. Suppose that (b) holds. Let $A^{\prime} \subset X^{\prime}$ and $B^{\prime} \subset Y^{\prime}$ be weak* closed and equicontinuous and let $\mu$ be a complex Borel measure on $A^{\prime} \times B^{\prime}$ such that

$$
z^{\prime}(x \otimes y)=\int_{A^{\prime} \times B^{\prime}}\left\langle x, x^{\prime}\right\rangle\left\langle y, y^{\prime}\right\rangle d \mu\left(x^{\prime}, y^{\prime}\right) \quad(x \in X, y \in Y) .
$$

Let $A_{1}^{\prime}$ denote the equicontinuous set $U_{S}{ }^{\prime}\left(A^{\prime}\right)$ and let $p_{A_{1}{ }^{\prime}}$ be the corresponding seminorm. If $w \in \mathcal{V}$ then, since $\mathcal{V}(y)$ is weak* metrizable, $w(y)$ is the weak ${ }^{*}$ limit of a sequence $\left\{V_{t_{n}} y\right\}$. The function $y^{\prime} \mapsto\left\langle y^{\prime}, w(y)\right\rangle$ is therefore Borel measurable on $B^{\prime}$, and by (b) of Lemma 4 and the dominated convergence theorem,

$$
\left\langle z^{\prime}, U_{s} x \otimes w(y)\right\rangle=\int_{A^{\prime} \times B^{\prime}}\left\langle U_{s} x, x^{\prime}\right\rangle\left\langle y^{\prime}, w(y)\right\rangle d \mu\left(x^{\prime}, y^{\prime}\right) \quad(s \in S) .
$$

Thus, for all $\alpha$ and all $s \in S$,

$$
\left|\left\langle z^{\prime}, U_{s} x \otimes\left(v_{\alpha}(y)-v(y)\right)\right\rangle\right| \leq p_{A_{1}{ }^{\prime}}(x) \int_{A^{\prime} \times B^{\prime}}\left|\left\langle y^{\prime}, v_{\alpha}(y)-v(y)\right\rangle\right| d|\mu|\left(x^{\prime}, y^{\prime}\right),
$$

so by (a) of Lemma 4

$$
\left|c_{\alpha}\right| \leq p_{A_{1}^{\prime}}(x) \int_{A^{\prime} \times B^{\prime}}\left|\left\langle y^{\prime}, v_{\alpha}(y)-v(y)\right\rangle\right| d|\mu|\left(x^{\prime}, y^{\prime}\right) .
$$

Since $\mathcal{V}(y)$ is weak ${ }^{*}$ metrizable, we may assume that $\left\{v_{\alpha}(y)\right\}$ is a sequence. The dominated convergence theorem then implies that $c_{\alpha} \rightarrow 0$. This completes the proof of the claim. 
It remains to prove (ii). Since $K(\mathcal{U}) \otimes K(\mathcal{V})$ is obviously an ideal of $\mathcal{W}, K(\mathcal{W}) \subset$ $K(\mathcal{U}) \otimes K(\mathcal{V})$. To show the reverse inclusion it suffices to show that if $d \in K(\mathcal{U})$ and $e \in K(\mathcal{V})$ are idempotents then $d \otimes e \in K(\mathcal{W})$. This follows easily from the fact that the idempotents in the minimal ideal are precisely those which are minimal with respect to the natural partial order on the set of all idempotents.

A similar theorem holds if $X$ is separable or if $U$ is almost periodic, provided one uses the left continuous tensor product.

By induction we have

Corollary 1. For $i=1,2, \ldots, n$ let $U^{i}$ be a weakly continuous, equicontinuous representation of a stsg $S_{i}$ on a lctvs $X_{i}$ such that one of the following holds:

(a) $U^{i}$ is weakly almost periodic for every $i$;

(b) for each $i \geq 2$, either $X_{i \beta}^{\prime}$ is separable or $U^{i}$ is almost periodic.

Then

(i) the tensor product representation $U_{\left(s_{1}, s_{2}, \ldots, s_{n}\right)}:=U_{s_{1}}^{1} \otimes U_{s_{2}}^{2} \otimes \cdots \otimes U_{s_{n}}^{n}$ is a weakly continuous, equicontinuous representation of $S_{1} \times S_{2} \times \cdots \times S_{n}$ on $X_{1} \otimes X_{2} \otimes \cdots \otimes X_{n}$ such that $\mathcal{U}=\mathcal{U}^{1} \otimes \mathcal{U}^{2} \otimes \cdots \otimes \mathcal{U}^{n}$ and $K(\mathcal{U})=$ $K\left(\mathcal{U}^{1}\right) \otimes K\left(\mathcal{U}^{2}\right) \otimes \cdots \otimes K\left(\mathcal{U}^{n}\right)$

(ii) if each $U^{i}$ is (weakly) almost periodic then $U$ is (weakly) almost periodic;

(iii) if $\mathcal{P}_{i}$ is a regular property of compactifications of $S_{i}$ and if $U_{i}$ is a $\mathcal{P}_{i}$ representation then the compactification $\mathcal{U}$ of $S_{1} \times S_{2} \times \cdots \times S_{n}$ is a factor of the direct product compactification $S_{1}^{\mathcal{P}_{1}} \times S_{2}^{\mathcal{P}_{2}} \times \cdots \times S_{n}^{\mathcal{P}_{n}}$; in particular,

(iv) if $\mathcal{P}$ is a regular property of compactifications which is inherited by finite direct products then $\bigotimes_{i=1}^{n} X_{i \mathcal{P}} \subset X_{\mathcal{P}}$.

We now prove an infinite tensor product version of Corollary 1 for commutative $C^{*}$-algebras. Let $\left\{X_{i}: i \in I\right\}$ be an arbitrary family of unital abelian $C^{*}$-algebras and let $I_{f}$ denote the collection of all nonempty finite subsets of $I$. The $C^{*}$-algebra tensor product $X:=\bigotimes_{i \in I} X_{i}$ is the $C^{*}$-algebra inductive limit of the family $\left\{Y_{a}:=\right.$ $\left.\bigotimes_{i \in a} X_{i}: a \in I_{f}\right\}$ relative to the maps $\Phi_{a, b}: Y_{b} \mapsto Y_{a}, b \subset a$, defined by $\Phi_{a, b}(y)=$ $y \otimes \bigotimes_{i \in a \backslash b} e_{i}$, where $e_{i}$ is the unit of $X_{i}$ [10, Section 1.23]. $X$ may be viewed as the closure of $Y:=\bigcup_{a \in I_{f}} Y_{a}$. For each $i \in I$ let $U^{i}$ be a representation of a stsg $S_{i}$ by unital*-homomorphisms on $X_{i}$. For $s=\left(s_{i}\right)_{i} \in S:=\prod_{i \in I} S_{i}$ define an operator $U_{s}$ on $Y$ by $\left.U_{s}\right|_{Y_{a}}=\bigotimes_{i \in a} U_{s_{i}}^{i}$. $U_{s}$ is well-defined and may be extended to an operator on $X=\bar{Y}$. It is easy to see that $U_{s}$ is a ${ }^{*}$-homomorphism and that $s \mapsto U_{s}$ is a unital representation of $S$ on $X$. $U$ is called the tensor product of the representations $U^{i}$ and is denoted by $\bigotimes_{i \in I} U^{i}$.

Corollary 2. For each $i \in I$ let $U^{i}$ be a weakly continuous representation of a stsg $S_{i}$ by unital *-homomorphisms on a unital abelian $C^{*}$-algebra $X_{i}$. Suppose that either

(a) $U^{i}$ is weakly almost periodic for every $i$, or

(b) for each $i$, either $X_{i \beta}^{\prime}$ is separable or $U^{i}$ is almost periodic.

Then

(i) $U:=\bigotimes_{i \in I} U^{i}$ is a weakly continuous representation of $S=\prod_{i \in I} S_{i}$ by unital * - homomorphisms on the $C^{*}$-algebra $X=\bigotimes_{i \in I} X_{i}$ such that $\mathcal{U}=$ $\lim _{a \in I_{f}} \bigotimes_{i \in a} \mathcal{U}^{i}$ and $K(\mathcal{U})=\lim _{a \in I_{f}} \bigotimes_{i \in a} K\left(\mathcal{U}^{i}\right)$;

(ii) if each $U^{i}$ is (weakly) almost periodic then $U$ is (weakly) almost periodic; 
(iii) if, for each $i, \mathcal{P}_{i}$ is a regular property of compactifications of $S_{i}$ and $U^{i}$ is a $\mathcal{P}_{i}$-representation, then $\mathcal{U}$ is a factor of the direct product compactification $\prod_{i \in I} S_{i}^{\mathcal{P}_{i}}$

(iv) if $\mathcal{P}$ is a regular property of compactifications which is inherited by finite direct products then $\bigotimes_{i \in I} X_{i \mathcal{P}} \subset X_{\mathcal{P}}$.

Proof. Since $X$ is the closure of $\bigcup_{a \in I_{f}} Y_{a}$, it follows from Corollary 1 that $U$ is weakly continuous and from Corollary 2 of Lemma 2 that $\mathcal{U} \cong \lim _{a \in I_{f}} \mathcal{U}_{Y_{a}}$. To complete the proof of part (i), use Corollary 1 and Lemma 2.

To prove (iii) note that by Corollary $1, \prod_{i \in I} S_{i}^{\mathcal{P}_{i}} \geq \prod_{i \in a} S_{i}^{\mathcal{P}_{i}} \geq \bigotimes_{i \in a} \mathcal{U}^{i}$ for all $a \in I_{f}$ hence, by Lemma 2 and part (i), $\prod_{i \in I} S_{i}^{\mathcal{P}_{i}} \geq \mathcal{U}$. Part (iv) follows from part (i) applied to the $C^{*}$-algebras $X_{i \mathcal{P}}$ and from Corollary 1 of Lemma 2.

Now assume that each $U^{i}$ is weakly almost periodic. Since $X$ is commutative, $X_{w a p}$ is a $C^{*}$-subalgebra of $X$. (For example, to see that $X_{w a p}$ is closed under multiplication, express $X$ as $C(\Omega)$ for some compact Hausdorff topological space $\Omega$ and use the fact that a norm bounded subset of $C(\Omega)$ is relatively compact in the weak topology if and only if it is relatively compact in the topology of pointwise convergence of $C(\Omega)$.) Since $X$ is generated by the $X_{i}$ and since $X_{i} \subset X_{\text {wap }}$, it follows that $X=X_{\text {wap }}$.

In the next section we show that the inclusion in part (iv) of Corollaries 1 and 2 may be proper (Example 8) but that equality holds for the right translation representation on certain subalgebras of $C\left(S_{i}\right)$ (Corollary to Theorem 8). The following result provides another instance of equality.

Corollary 3. Suppose that, in addition to the hypotheses of Corollary 2, WAP( $\left.S_{i}\right)$ is left amenable for each $i$. Then $X_{\text {sap }}=\bigotimes_{i} X_{i \text { sap }}$ and $X$ has the splitting property (see Example 6).

Proof. Let $V$ and $W$ be weakly continuous, weakly almost periodic, equicontinuous representations of stsgs $S$ and $T$ on lctvs $Y$ and $Z$, respectively, such that $Y=Y_{u m r i}$ and $Z=Z_{\text {umri }}$. By Corollary $1, Y \otimes Z=(Y \otimes Z)_{\text {umri }}$ and $Y_{\text {sap }} \otimes Z_{\text {sap }} \subset(Y \otimes Z)_{\text {sap }}$. Let $x=\lim _{n} \sum_{i} y_{\text {in }} \otimes z_{i n} \in(Y \otimes Z)_{\text {sap }}$, where $y_{\text {in }} \in Y$ and $z_{\text {in }} \in Z$, and let $d \in \mathcal{V}$ and $e \in \mathcal{W}$ be minimal idempotents. Then $d \otimes e$ is a minimal idempotent in $\mathcal{V} \otimes \mathcal{W}$, and by Example $6 x=(d \otimes e) x=\lim _{n} \sum_{i} d\left(y_{i n}\right) \otimes e\left(z_{i n}\right) \in Y_{m d} \otimes Z_{m d}=Y_{\text {sap }} \otimes Z_{\text {sap }}$. Thus, $(Y \otimes Z)_{\text {sap }}=Y_{\text {sap }} \otimes Z_{\text {sap }}$.

Now, since $W A P\left(S_{i}\right)$ is left amenable, $\mathcal{U}^{i}$ has unique minimal right ideal [2, 6.2.14], [3]. Therefore, by Corollary $2, X=X_{\text {umri }}$ hence $X$ has the splitting property. By the same corollary, $\bigotimes_{i} X_{i s a p} \subset X_{\text {sap }}$. For the reverse inclusion, let $x=\lim _{n} y_{n} \in X_{\text {sap }}$, where $y_{n} \in Y_{n}:=\bigotimes_{i \in a_{n}} X_{i}, a_{n} \in I_{f}$. By the first part of the proof, $Y_{n s a p}=\bigotimes_{i \in a_{n}} X_{i \text { sap }}$. Let $e$ be any minimal idempotent in $\mathcal{U}$. Then $x=e(x)=\lim _{n} e\left(y_{n}\right)$ and $e\left(y_{n}\right) \in Y_{n \text { sap }}$ so $x \in \bigotimes_{i} X_{i \text { sap }}$.

\section{Compactifications of Infinite Direct Products of Semigroups}

Let $\left\{S_{i}: i \in I\right\}$ be an infinite family of stsgs with identity. In this section we consider tensor products of right m-introverted $C^{*}$-subalgebras of $C\left(S_{i}\right)$ by examining compactifications of the direct product semigroup $S:=\prod_{i \in I} S_{i}$. The following notion is fundamental in the construction of such compactifications. 
Definition 2. A left topological compactification $\left(\varphi, T^{\prime}\right)$ of a stsg $T$ with identity is said to have the local joint continuity property if the mapping

$$
\left(t^{\prime}, t\right) \mapsto t^{\prime} \varphi(t): T^{\prime} \times T \mapsto T^{\prime}
$$

is continuous at each point of $T^{\prime} \times \varphi(1)$.

For example, if $T^{\prime}$ is semitopological or if $T$ is locally compact or complete metrizable, then, by a theorem of Lawson $[9],\left(\varphi, T^{\prime}\right)$ has the local joint continuity property. The local joint continuity property is clearly inherited by factors, direct products, and subcompactifications.

We shall use the following notation: For each $a \subset I$ let $S_{a}:=\prod_{i \in a} S_{i}$ and let $p_{a}: S \mapsto S_{a}$ and $q_{a}: S_{a} \mapsto S$ denote the projection and canonical injection mappings. We set $r_{a}:=q_{a} \circ p_{a}$. The directed set of all nonempty finite subsets of $I$ is denoted by $I_{f}$.

Lemma 7. Let $\left(\varphi, S^{\prime}\right)$ be a left topological compactification of $S$. If $S$ has the local joint continuity property then $\varphi(s)=\lim _{a \in I_{f}} \varphi\left(r_{a}(s)\right)$ uniformly in $s \in S$ (relative to the unique uniformity on $S^{\prime}$ compatible with the topology).

Proof. A standard compactness argument shows that if $O$ is a neighborhood of the diagonal in $S^{\prime} \times S^{\prime}$ then there exists a neighborhood $N$ of the identity in $S$ such that

$$
\left(s^{\prime} \varphi(s), s^{\prime}\right) \in O \quad\left(s \in N, s^{\prime} \in S^{\prime}\right) .
$$

Now choose $a \in I_{f}$ and a neighborhood $N_{a}$ of the identity in $S_{a}$ such that $p_{a}^{-1}\left(N_{a}\right) \subset$ $N$. For any $s \in S$ and $b \in I_{f}$ with $b \supset a$ we have $r_{I \backslash b}(s) \in p_{a}^{-1}\left(N_{a}\right)$ and $r_{b}(s)$. $r_{I \backslash b}(s)=s$ hence

$$
\left(\varphi(s), \varphi\left(r_{b}(s)\right)\right)=\left(\varphi\left(r_{b}(s)\right) \cdot \varphi\left(r_{I \backslash b}(s)\right), \varphi\left(r_{b}(s)\right)\right) \in O .
$$

Definition 3. Let $\mathcal{P}_{i}$ be a regular property of semigroup compactifications of $S_{i}$, $i \in I$. A compactification $\left(\varphi, S^{\prime}\right)$ of $S$ will be called a $\mathcal{P}_{I^{-}}$compactification if it satisfies the following conditions:

(a) For each $i \in I$, the subcompactification $\left(\varphi_{i}, S_{i}^{\prime}\right):=\left(\varphi \circ q_{i}, \overline{\varphi \circ q_{i}\left(S_{i}\right)}\right)$ of $S^{\prime}$ is a $\mathcal{P}_{i}$-compactification of $S_{i}$.

(b) For each $a \in I_{f}$ and each choice of $s_{i}^{\prime} \in S_{i}^{\prime}$, the set $\left\{s_{i}^{\prime}: i \in a\right\}$ is commutative and the multiplication map $\mu_{a}^{\prime}: S_{a}^{\prime}:=\prod_{i \in a} S_{i}^{\prime} \mapsto S^{\prime}, \mu_{a}^{\prime}\left(\left(s_{i}^{\prime}\right)_{i \in a}\right)=\prod_{i \in a} s_{i}^{\prime}$, is continuous.

(c) $\varphi(s)=\lim _{a \in I_{f}} \varphi\left(r_{a}(s)\right)$ uniformly in $s \in S$.

Note that the commutativity condition in (b) always holds for elements $s_{i}^{\prime}=$ $\varphi\left(q_{i}\left(s_{i}\right)\right)$. Hence, if $\left(\varphi, S^{\prime}\right)$ is a semitopological compactification of $S$ then the commutativity condition holds for arbitrary $s_{i}^{\prime} \in S_{i}^{\prime}$.

Lemma 8. Let $\mathcal{P}_{i}$ be a regular property of semigroup compactifications of $S_{i}$ and let $\left(\psi_{i}, S_{i}^{\mathcal{P}_{i}}\right)$ be the universal $\mathcal{P}_{i}$-compactification of $S_{i}, i \in I$. If each $\left(\psi_{i}, S_{i}^{\mathcal{P}_{i}}\right)$ has the local joint continuity property then $\mathcal{P}_{I}$ is regular and the direct product compactification

$$
(\psi, \tilde{S}):=\left(\prod_{i \in I} \psi_{i}, \prod_{i \in I} S_{i}^{\mathcal{P}_{i}}\right)
$$

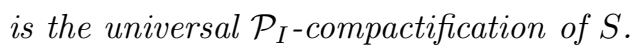


Proof. Denote the projection and canonical injection mappings by $\tilde{p}_{a}: \tilde{S} \mapsto \tilde{S}_{a}$ and $\tilde{q}_{a}: \tilde{S}_{a} \mapsto \tilde{S}$, respectively. Let $\left(\varphi, S^{\prime}\right)$ be a factor of $(\psi, \tilde{S})$ with compactification homomorphism $\theta:(\psi, \tilde{S}) \mapsto\left(\varphi, S^{\prime}\right)$. We show that $\left(\varphi, S^{\prime}\right)$ is a $\mathcal{P}_{I^{-} \text {-compactification }}$ of $S$. Let $\theta_{i}:=\theta \circ \tilde{q}_{i}: S_{i}^{\mathcal{P}_{i}} \mapsto S^{\prime}$. Then $\theta_{i} \circ \psi_{i}=\theta \circ \tilde{q}_{i} \circ \psi_{i}=\theta \circ \psi \circ q_{i}=\varphi \circ q_{i}$, so $S_{i}^{\prime}=\theta_{i}\left(S_{i}^{\mathcal{P}_{i}}\right)$. Since $\mathcal{P}_{i}$ is regular, $S_{i}^{\prime}$ is a $\mathcal{P}_{i}$-compactification of $S_{i}$. Therefore, $\left(\varphi, S^{\prime}\right)$ satisfies (a) of Definition 3. To verify (b), let $a \in I_{f}$ and note that the multiplication map

$$
\tilde{\mu}_{a}: \tilde{S}_{a} \mapsto \tilde{S},\left(\tilde{s}_{i}\right)_{i \in a} \mapsto \prod_{i \in a} \tilde{q}_{i}\left(\tilde{s}_{i}\right)
$$

is well defined and continuous. Since $\left\{\theta_{i}\left(\tilde{s}_{i}\right)=\theta \circ \tilde{q}_{i}\left(\tilde{s}_{i}\right): i \in a\right\}$ is commutative and $\theta \circ \tilde{\mu}_{a}\left(\left(\tilde{s}_{i}\right)_{i \in a}\right)=\mu_{a}^{\prime}\left(\left(\theta_{i}\left(\tilde{s}_{i}\right)\right)_{i \in a}\right)$, it follows that $\mu_{a}^{\prime}$ is well defined and continuous. To verify (c) of Definition 3 use the fact that $S^{\prime}$ has the local joint continuity property (because $\tilde{S}$ does) and apply Lemma 7 .

We have shown that any factor of $(\psi, \tilde{S})$ is a $\mathcal{P}_{I}$-compactification of $S$. To complete the proof of the lemma we need to verify that the converse holds. So let

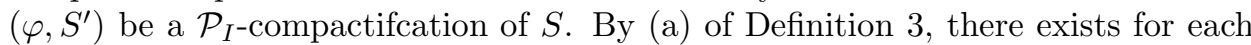
$i \in I$ a continuous homomorphism $\theta_{i}: S_{i}^{\mathcal{P}_{i}} \mapsto S_{i}^{\prime}$ such that $\theta_{i} \circ \psi_{i}=\varphi \circ q_{i}$. For $a \in I_{f}$ let $\left(\psi_{a}, \tilde{S}_{a}\right):=\left(\prod_{i \in a} \psi_{i}, \prod_{i \in a} S_{i}^{\mathcal{P}_{i}}\right)$ and define $\theta_{a}: \tilde{S}_{a} \mapsto S^{\prime}$ by

$$
\theta_{a}\left(\left(u_{i}\right)_{i \in a}\right)=\mu_{a}^{\prime}\left(\left(\theta_{i}\left(u_{i}\right)\right)_{i \in a}\right)
$$

By (b) of Definition 3, $\theta_{a}$ is well defined and continuous. Also, from $\theta_{i} \circ \psi_{i}=\varphi \circ q_{i}$ we have $\theta_{a} \circ \psi_{a}=\varphi \circ q_{a}$, so $\theta_{a}$ is a homomorphism on a dense subsemigroup of $\tilde{S}_{a}$ and hence on $\tilde{S}_{a}$. By (c) of Definition 3 , if $W$ is a closed neighborhood of the diagonal in $S^{\prime} \times S^{\prime}$, then for some $a \in I_{f}$ and any $b, c \in I_{f}$ containing $a$,

$$
\left(\theta_{b} \circ \tilde{p}_{b} \circ \psi(s), \theta_{c} \circ \tilde{p}_{c} \circ \psi(s)\right)=\left(\varphi \circ r_{b}(s), \varphi \circ r_{c}(s)\right) \in W \quad(s \in S) .
$$

(Here we have used the identities $\tilde{p}_{b} \circ \psi=\psi_{b} \circ p_{b}$ and $\theta_{b} \circ \psi_{b}=\varphi \circ q_{b}, b \in I_{f}$.) It follows that $\left(\theta_{b} \circ \tilde{p}_{b}(u), \theta_{c} \circ \tilde{p}_{c}(u)\right) \in W$ for all $u \in \tilde{S}$. Therefore, $\left\{\theta_{b} \circ \tilde{p}_{b}: b \in I_{f}\right\}$ is a uniform Cauchy net, so the limit

$$
\theta(u):=\lim _{b} \theta_{b} \circ \tilde{p}_{b}(u)=\lim _{b} \mu_{b}^{\prime}\left(\left(\theta_{i}\left(u_{i}\right)\right)_{i \in b}\right) \quad(u \in \tilde{S})
$$

exists and defines a continuous mapping $\theta: \tilde{S} \mapsto S^{\prime}$ such that $\theta \circ \psi=\varphi$. Therefore $S^{\prime}$ is a factor of $\tilde{S}$.

The next theorem gives sufficient conditions for a factor of the direct product compactification $(\psi, \tilde{S})$ to be a direct product of compactifications.

Theorem 7. For each $i \in I$ let $\mathcal{P}_{i}$ be a regular property of semigroup compactifications of $S_{i}$ and let $S_{i}^{\mathcal{P}_{i}}$ have the local joint continuity property. If a factor $\left(\varphi, S^{\prime}\right)$ of $(\psi, \tilde{S})$ is universal with respect to a regular property $\mathcal{P}$ which is inherited by subcompacifications, then $S^{\prime}$ is isomorphic to a direct product of $\mathcal{P}$-compactifications. If $\mathcal{P}$ is also inherited by direct products then $S^{\prime} \cong \prod_{i \in I} S_{i}^{\mathcal{P}}$.

Proof. We show that $\left(\varphi, S^{\prime}\right) \cong\left(\prod_{i \in I} \varphi_{i}, \prod_{i \in I} S_{i}^{\prime}\right)$ (notation as in (a) of Definition $3)$. Let $\theta:(\psi, \tilde{S}) \mapsto\left(\varphi, S^{\prime}\right)$ be thecompactification homomorphism. Then $\theta_{i}:=\theta \circ$ 
$\tilde{q}_{i}:\left(\psi_{i}, S_{i}^{\mathcal{P}_{i}}\right) \mapsto\left(\varphi_{i}, S_{i}^{\prime}\right)$ is a compactification homomorphism, where $\tilde{q}_{i}: S_{i}^{\mathcal{P}_{i}} \mapsto \tilde{S}$ is the canonical injection. Define a mapping $\Psi: S^{\prime} \mapsto \prod_{i \in I} S_{i}^{\prime}$ by $\Psi(\theta(u))=\left(\theta_{i}\left(u_{i}\right)\right)_{i}$, where $u=\left(u_{i}\right)_{i} \in \tilde{S}$. To see that $\Psi$ is well defined, consider the compactification $\left(\varphi \circ r_{i}, \overline{\varphi \circ r_{i}(S)}\right)$ of $S$, which is a subcompactification of $S^{\prime}$ and hence, by hypothesis, a $\mathcal{P}$-compactification. Thus there exists a continuous mapping $\phi_{i}: S^{\prime} \mapsto S^{\prime}$ such that $\phi_{i} \circ \varphi=\varphi \circ r_{i}$. If $\tilde{p}_{i}: \tilde{S} \mapsto S_{i}^{\mathcal{P}_{i}}$ denotes the projection mapping then $\phi_{i} \circ$ $\theta(\psi(s))=\phi_{i} \circ \varphi(s)=\varphi \circ r_{i}(s)=\varphi \circ q_{i}\left(s_{i}\right)=\theta_{i} \circ \psi_{i}\left(s_{i}\right)=\theta_{i} \circ \tilde{p}_{i}(\psi(s))$, hence $\phi_{i} \circ \theta(u)=\theta_{i}\left(u_{i}\right)$. This shows that $\Psi$ is well defined and continuous. By (13), $\Psi$ is also $1-1$ and hence is a compactification isomorphism. Since $S^{\prime} \geq S_{i}^{\prime}, S_{i}^{\prime}$ is a $\mathcal{P}$-compactification.

Finally, if $\mathcal{P}$ is inherited by direct products then $\prod_{i \in I} S_{i}^{\mathcal{P}}$ is a $\mathcal{P}$-compactification of $S$ so $\prod_{i \in I} S_{i}^{\prime} \geq \prod_{i \in I} S_{i}^{\mathcal{P}}$ and hence $S_{i}^{\prime} \cong S_{i \mathcal{P}}$.

The next theorem, which generalizes results of [4] and [8], gives sufficient conditions for a compactification of $S$ to be a direct product of compactifications.

Theorem 8. Let $\mathcal{P}_{i}=\mathcal{P}$ for all $i$, where $\mathcal{P}$ is a regular property of compactifications which is inherited by subcompactifications and direct products. If $\mathcal{P}$ compactifications are topological then $S^{\mathcal{P}_{I}} \cong S^{\mathcal{P}}$.

Proof. Since $\mathcal{P}$ is inherited by direct products, $S^{\mathcal{P}_{I}}(=\tilde{S})$ is a $\mathcal{P}$-compactification

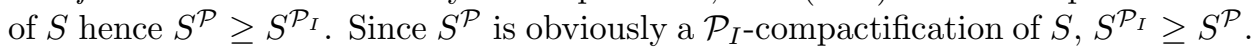

Corollary. Let $X_{i}$ be a $C^{*}$-subalgebra of $W A P\left(S_{i}\right)$ containing $A P\left(S_{i}\right)$ and let $X$ be the $C^{*}$-algebra tensor product $\bigotimes_{i \in I} X_{i}$. If $\mathcal{P}$ is a regular property inherited by subcompacifications and direct products and if $\mathcal{P}$ compactifications are topological then, with respect to the right translation representation, $X_{\mathcal{P}}=\bigotimes_{i \in I} X_{i \mathcal{P}}$.

Proof. By Theorem $2, X_{\mathcal{P}}$ is a unital $C^{*}$-subalgebra of $X \subset W A P(S)$. Also, since $L_{s} \circ R_{t}=R_{t} \circ L_{s}$ it follows from the corollary to Lemma 3 that $L_{S} X_{\mathcal{P}} \subset X_{\mathcal{P}}$. Therefore $X_{\mathcal{P}}$ is left and right introverted $[2,4.2 .7]$ so we may form the canonical $X_{\mathcal{P}}$-compactification $S^{X_{\mathcal{P}}}$. Since $S$ has an identity, $S^{X_{\mathcal{P}}} \cong \mathcal{R}_{X_{\mathcal{P}}}$ (Example 1). We claim that $X_{\mathcal{P}}=\varphi^{*}\left(C\left(S^{\mathcal{P}}\right)\right)$. Indeed, since $\mathcal{P}$-compactifications are topological, $Y:=\varphi^{*}\left(C\left(S^{\mathcal{P}}\right)\right) \subset A P(S)=\bigotimes_{i} A P\left(S_{i}\right) \subset X$, and since $R_{Y} \cong S^{\mathcal{P}}, Y \subset X_{\mathcal{P}}$. On the other hand, since $S^{X_{\mathcal{P}}} \cong \mathcal{R}_{X_{\mathcal{P}}}, S^{X_{\mathcal{P}}}$ has property $\mathcal{P}$ hence $X_{\mathcal{P}} \subset Y$. Therefore, $X_{\mathcal{P}}=\varphi^{*}\left(C\left(S^{\mathcal{P}}\right)\right)$, as claimed. Similarly, $X_{i \mathcal{P}}=\varphi^{*}\left(C\left(S_{i}^{\mathcal{P}}\right)\right)$. Since $S^{\mathcal{P}}=\prod_{i} S_{i}^{\mathcal{P}}$ (Theorem 8), $X_{\mathcal{P}}=\varphi^{*}\left(C\left(S^{\mathcal{P}}\right)\right)=\bigotimes_{i} \varphi_{i}^{*}\left(C\left(S_{i}^{\mathcal{P}}\right)\right)=\bigotimes_{i} X_{i \mathcal{P}}$.

The conclusions of Theorem 7 , Theorem 8 and the above corollary hold if $\mathcal{P}$ is any of the following properties of compactifications $\left(\varphi, T^{\prime}\right): T^{\prime}$ is a topological group; $T^{\prime}$ is a topological semilattice; $T^{\prime}$ is a left simple, right simple, or simple topological semigroup [2, p. 32]; $T^{\prime}$ is a left zero or right zero semigroup; more generally, $T^{\prime}$ is a topological semigroup which satisfies a family of identities $[2$, p. 119].

The conclusions of Theorems 7 and 8 fail in general if $\mathcal{P}$ is not inherited by subcompactifications, as the following example demonstrates.

Example 8. Let $\mathcal{P}$ be the property of compactifications $\left(\varphi, S^{\prime}\right)$ of locally compact stsgs $S$ that multiplication in $S^{\prime}$ is jointly continuous and $\lim _{s \rightarrow \infty} \varphi(s)$ exists. If $T$ is the interval $[0, \infty)$ under addition, then $\left(\varphi,(T \times T)^{\mathcal{P}}\right) \cong\left(\epsilon,(T \times T)^{F}\right)$, where $F$ is the $\mathrm{C}^{*}$-algebra of almost periodic functions $f$ on $T \times T$ with the property that 
$\lim _{(s, t) \rightarrow(\infty, \infty)} f(s, t)$ exists. Similarly, $T^{\mathcal{P}} \times T^{\mathcal{P}}=T^{\infty} \times T^{\infty} \cong(T \times T)^{G}$, where $G$ is contained in the $\mathrm{C}^{*}$-algebra of almost periodic functions $f$ on $T \times T$ with the property that $\lim _{s \rightarrow \infty} \lim _{t \rightarrow \infty} f(s, t)$ and $\lim _{t \rightarrow \infty} \lim _{s \rightarrow \infty} f(s, t)$ exist. Since there are almost periodic functions $f$ on $T \times T$ for which the joint limit exists but the iterated limits do not (e.g., $\left.f(s, t)=e^{i(s+t)}\left[(s+1)^{-1}+(t+1)^{-1}\right]\right),(T \times T)^{\mathcal{P}}>T^{\mathcal{P}} \times T^{\mathcal{P}}$. Therefore $(T \times T)^{P}$ is not a direct product of compactifications, hence the conclusion of Theorem 8 fails. Note that $(T \times T)^{A P} \cong T^{A P} \times T^{A P}>(T \times T)^{\mathcal{P}}$, hence the conclusion of Theorem 7 also fails, as does that of the above corollary.

\section{REFERENCES}

1. J.F. Berglund, H.D. Junghenn and P. Milnes, Compact Right Topological Semigroups and Generalizations of Almost Periodicity, Lecture Notes in Mathematics 663, Springer-Verlag, New York, 1978. MR 80c:22003

2. __ Analysis on Semigroups: Function Spaces, Compactifications, Representations, Wiley, New York, 1989. MR 91b:43001

3. K. de Leeuw and I. Glicksberg, Applications of almost periodic compactifications, Acta Math. 105 (1961), 63-97. MR 24:A1632

4. - Almost periodic functions on semigroups, Acta Math. 105 (1961), 99-140. MR 24:A1633

5. R. Ellis, Distal transformation groups, Pacific J. Math. 9 (1958), 401-405. MR 21:96

6. L_Lectures on Topological Dynamics, Benjamin, New York, 1969. MR 42:2463

7. H.D. Junghenn, Tensor products and almost periodicity, Proc. Amer. Math. Soc. 43 (1974), 99-105. MR 51:1476

8. $-C^{*}$-algebras of functions on direct products of semigroups, Rocky Mountain J. Math. 10 (1980), 589-597. MR 81m:22004

9. J.D. Lawson, Joint continuity in semitopological semigroups, Illinois J. Math. 18 (1974) 275-285. MR 49:454

10. S. Sakai, $C^{*}$-Algebras and $W^{*}$-Algebras, Springer-Verlag, New York, 1971. MR 56:1082

11. H. H. Schaefer, Topological Vector Spaces, Springer-Verlag, New York, 1971. MR 49:7722

12. K. Witz, Applications of a compactification for bounded operator semigroups, Illinois J. Math. 8 (1964), 685-696. MR 31:2626

Department of Mathematics, The George Washington University, Washington, D.C. 20052

E-mail address: hugo@math.gwu.edu 